\title{
Model approximation using magnitude and phase criteria: Implications for model reduction and system identification
}

\author{
Henrik Sandberg ${ }^{1, *}{ }^{\dagger}$, Alexander Lanzon ${ }^{2,3}$ and Brian D. O. Anderson ${ }^{2,3}$ \\ ${ }^{1}$ California Institute of Technology, Control and Dynamical Systems, M/C 107-81, Pasadena, \\ CA 91125, U.S.A. \\ ${ }^{2}$ Research School of Information Sciences and Engineering, The Australian National University, \\ Canberra ACT 0200, Australia \\ ${ }^{3}$ National ICT Australia Ltd., Locked Bag 8001, Canberra ACT 2601, Australia
}

\begin{abstract}
SUMMARY
In this paper, we use convex optimization for model reduction and identification of transfer functions. Two different approximation criteria are studied. When the first criterion is used, magnitude functions are matched, and when the second criterion is used, phase functions are matched. The weighted error bounds have direct interpretation in a Bode diagram, and are suitable to engineers working with frequency-domain data. We also show that transfer functions that have similar magnitude or phase functions have a small relative $\mathrm{H}$-infinity error, under certain stability and minimum phase assumptions. The error bounds come from bounds associated with the Hilbert transform operator restricted in its application to rational transfer functions. Furthermore, it is shown how the approximation procedures can be implemented with linear matrix inequalities, and four examples are included to illustrate the results. Copyright (C) 2006 John Wiley \& Sons, Ltd.
\end{abstract}

Received 8 December 2005; Revised 15 April 2006; Accepted 4 September 2006

KEY WORDS: model approximation; model reduction; system identification; semidefinite programs

\section{INTRODUCTION}

It has become increasingly popular in control and systems theory to use convex optimization to solve problems of various nature, see, for example, $[1,2]$. One reason is the efficient software

\footnotetext{
*Correspondence to: Henrik Sandberg, California Institute of Technology, Control and Dynamical Systems, M/C 107-81, Pasadena, CA 91125, U.S.A.

${ }^{\dagger}$ E-mail: henriks@cds.caltech.edu

Contract/grant sponsor: Australian Research Council; contract/grant number: DP0342683

Contract/grant sponsor: National ICT Australia Ltd.
} 
available for solving semidefinite programs in polynomial time. Another reason is that many well-known problems, such as $H_{\infty}$ optimization, can be cast as semidefinite programs using linear matrix inequalities (LMIs). It is, however, not known how to use these tools to obtain optimal transfer function approximations in the $H_{\infty}$ norm. The reason is a non-convex rank constraint that is needed to enforce the degree of the approximation [2, Corollary 7.11]. For this reason, various suboptimal methods, such as balanced truncation [2-4], are often being used for model reduction.

One possibility for obtaining an optimal model reduction problem that can be solved in polynomial time is to change the norm. The Hankel norm model reduction problem can be solved in polynomial time, and often yields good approximations in $H_{\infty}$ norm as well, see [5]. Other new interesting approaches are to use the real part norm, see [6], or a special parametrization of unstable transfer functions together with the $L_{\infty}$ norm, see [7]. In particular, the methods in [6,7] give tighter lower bounds on the approximation error than Hankel approximation, easily incorporate weights, and can be used with samples of frequency data or the impulse response. This opens up the possibility of using the methods in $[6,7]$ for system identification as well.

In this paper, we show that transfer functions of given degree can be matched optimally to magnitude and phase data in polynomial time, using standard LMI solvers. The proposed methods share some advantages with the methods in [6,7], such as the possibility of using weights and samples of data, but also have direct interpretations in Bode diagrams. Two examples of the problems we solve (in the single-input single-output (SISO) case for ease of illustration) are

$$
\begin{gathered}
\left\|\log \left|G_{\mathrm{a}}\right|-\log |G|\right\|_{\infty} \rightarrow \min \\
\left\|\arg G_{\mathrm{a}}-\arg G\right\|_{\infty} \rightarrow \min
\end{gathered}
$$

where $G$ is a given transfer function, and $G_{\mathrm{a}}$ is a stable approximation of user-specified degree. Since we match either magnitude or phase, we must put additional assumptions on $G_{\mathrm{a}}$ to obtain unique solutions. Generally, we will require $G_{\text {a }}$ to be minimum phase and stable. This should be the situation of most interest. However, $G_{\mathrm{a}}$ can easily be modified to include unstable poles or zeroes. Two other examples of the problems we solve (in the SISO case for ease of illustration) are to find $G_{\mathrm{a}}$ such that

$$
\begin{gathered}
\log \left|w_{2}(\mathrm{j} \omega)\right| \leqslant \log \left|G_{\mathrm{a}}(\mathrm{j} \omega)\right|-\log |G(\mathrm{j} \omega)| \leqslant-\log \left|w_{1}(\mathrm{j} \omega)\right| \\
\arg w_{2}(\mathrm{j} \omega) \leqslant \arg G_{\mathrm{a}}(\mathrm{j} \omega)-\arg G(\mathrm{j} \omega) \leqslant-\arg w_{1}(\mathrm{j} \omega)
\end{gathered}
$$

for all $\omega$, where $w_{1}, w_{2}$ are user-specified weights. In (3) it is assumed that $\left\|w_{1}\right\|_{\infty} \leqslant 1$ and $\left\|w_{2}\right\|_{\infty} \leqslant 1$, and in (4) it is assumed that $\arg w_{1} \leqslant 0$ and $\arg w_{2} \leqslant 0$. For the magnitude approximation case, we will be able to deal with multi-input multi-output (MIMO) systems as well. For the phase approximation case, we will not deal with MIMO systems. One reason for this is the splintering of definition of what phase is in the MIMO case, see [8-10]. Furthermore, we obtain a priori bounds on the relative error in the $H_{\infty}$ norm, $\left\|G^{-1}\left(G-G_{\mathrm{a}}\right)\right\|_{\infty}$. Many other methods, such as optimal Hankel norm and real part norm approximation, give a priori bounds on the additive error $\left\|G-G_{\mathrm{a}}\right\|_{\infty}$. To go from an additive bound to a relative bound using just the submultiplicative property of the $H_{\infty}$ norm is generally too conservative. When reducing 
plant models that should be used in feedback control design, the relative error is often a better reduction criterion, see [4, p. 101].

To illustrate the methods developed here, four examples are included. They show how magnitude and phase approximation can be used for model reduction, system identification, and filter design in Bode diagrams. Also, a comparison with [7] is included. Hence, the results should be of interest to both control and signal processing engineers.

The organization of the paper is as follows. In Section 2, we formulate and solve the magnitude approximation problem. In particular, how the problem is formulated as a semidefinite program is explained in Section 2.1. In Section 3, we formulate and solve the phase approximation problem. The details of the semidefinite program are given in Section 3.1. The methods are illustrated in Section 4, where four different examples are given. Finally, in Section 5 we have conclusions and suggestions for future work. Some of the results in this paper are given without proof in the shorter conference paper [11].

\section{Preliminaries}

The modulus on $\mathbb{C}$ is denoted by $|\cdot|$. The $p$ singular values of a $p \times p$ complex matrix $M$ are denoted by $\sigma_{i}(M), i=1 \ldots p$, in decreasing order. The maximum and minimum singular values are also denoted by $\bar{\sigma}(M)$ and $\sigma(M)$, respectively. $M^{*}$ is the complex conjugate transpose of $M$. The $L_{\infty}$ norm $\|\cdot\|_{\infty}$ of a $p \times p$ matrix function $G(s)$ of a complex variable $s$ is defined by $\|G\|_{\infty}:=\operatorname{ess}_{\sup _{\omega}} \bar{\sigma}(G(\mathrm{j} \omega))$ and $G \in L_{\infty}$ if $\|G\|_{\infty}$ is finite. A matrix function $G \in H_{\infty}$ if it is bounded and analytic in the open complex right half plane $\left(\mathbb{C}_{+}\right)$, and $G \in H_{\infty}^{-}$if it is bounded and analytic in the open left half plane $\left(\mathbb{C}_{-}\right) . G \in R H_{\infty}\left(R L_{\infty}\right)$ if it is in $H_{\infty}\left(L_{\infty}\right)$ and is rational in $s$ with real coefficients. Furthermore, $G \in R_{n} H_{\infty}$ if $G \in R H_{\infty}$ and has McMillan degree less than or equal to $n$. The conjugate system $G^{\sim}$ is defined by $G^{\sim}(s):=G(-s)^{\mathrm{T}}$ (and $G(\mathrm{j} \omega)^{*}=G^{\sim}(\mathrm{j} \omega)$ if $G$ has real coefficients). A square system $G$ is all-pass if $G(\mathrm{j} \omega)^{*} G(\mathrm{j} \omega)=I$. We call a matrix function $G$ minimum phase if it has full rank in $\mathbb{C}_{+}$, and stable if it belongs to $H_{\infty}$.

\section{MAGNITUDE APPROXIMATION}

In this section, we formulate an optimization problem that allow us to approximate a transfer function $G$ with another transfer function $G_{\mathrm{a}}$ (of smaller degree) such that the gains $\sigma_{i}(G(\mathrm{j} \omega))$ and $\sigma_{i}\left(G_{\mathrm{a}}(\mathrm{j} \omega)\right)$ are close. The following assumptions on the model $G$ and the weights $w_{1}$, $w_{2}$ will be used for magnitude approximation:

Assumption 1

$G, G^{-1}, w_{1}, w_{1}^{-1}, w_{2}, w_{2}^{-1} \in R L_{\infty}, w_{1}, w_{2}$ are scalar, and $\left\|w_{1}\right\|_{\infty} \leqslant 1,\left\|w_{2}\right\|_{\infty} \leqslant 1$.

Remark 1 (Strictly proper models)

The condition $G^{-1} \in R L_{\infty}$ in Assumption 1 excludes strictly proper models $G$ and $G$ with zeroes on the imaginary axis. The condition $G^{-1} \in R L_{\infty}$ can be relaxed, however. For example, one can instead require that there is a positive integer $n$ such that $\lim _{s \rightarrow \infty} \operatorname{det}\left(s^{n} G(s)\right) \neq 0$. The reason for the conditions on $G$ is to easily guarantee that there exists a minimum phase spectral factor in $R_{m p} H_{\infty}$. 
The optimization problem we shall try to solve is: given a $p \times p$ MIMO transfer function $G$ and weights $w_{1}, w_{2}$ that satisfy Assumption 1, and a positive integer $m$, find $\gamma$ and an approximation $G_{\mathrm{a}}$ such that

$$
\begin{aligned}
& \min _{\gamma, G_{\mathrm{a}}} \gamma \quad \text { s.t. } \\
& \frac{\left|w_{2}(\mathrm{j} \omega)\right|}{\sqrt{1+\gamma}} \leqslant \sigma_{i}\left(\left(G^{-1} G_{\mathrm{a}}\right)(\mathrm{j} \omega)\right) \leqslant \frac{\sqrt{1+\gamma}}{\left|w_{1}(\mathrm{j} \omega)\right|} \text { for all } \omega \text { and } i=1 \ldots p \\
& \gamma \geqslant 0 \quad \text { and } \quad G_{\mathrm{a}}, G_{\mathrm{a}}^{-1} \in R_{m} H_{\infty}
\end{aligned}
$$

As we shall see in this section, in the SISO case $(p=1)$, problem (5)-(7) is a quasiconvex minimization problem (see [12, p. 145]) and we will find the optimal approximation $G_{\mathrm{a}}$ in $R_{m} H_{\infty}$. In the MIMO case ( $\left.p>1\right)$, we will find a solution if we allow $G_{\text {a }}$ to belong to a set larger than $R_{m} H_{\infty}$. This set contains $R_{m} H_{\infty}$ and is a subset of $R_{m p} H_{\infty}$. Next, we motivate why it is interesting to find solutions to (5)-(7).

The constraints (6) can be reformulated in a number of ways, as is seen in the following theorem.

\section{Theorem 1}

Assume that $w_{1}, w_{2}$ have no zeroes on the imaginary axis. Then (6) is equivalent to (8) and (9) separately

$$
\begin{gathered}
\frac{\left|w_{1}(\mathrm{j} \omega)\right|}{\sqrt{1+\gamma}} \leqslant \sigma_{i}\left(\left(G_{\mathrm{a}}^{-1} G\right)(\mathrm{j} \omega)\right) \leqslant \frac{\sqrt{1+\gamma}}{\left|w_{2}(\mathrm{j} \omega)\right|} \\
\left\|w_{1} G^{-1} G_{\mathrm{a}}\right\|_{\infty} \leqslant \sqrt{1+\gamma} \text { and }\left\|w_{2} G_{\mathrm{a}}^{-1} G\right\|_{\infty} \leqslant \sqrt{1+\gamma}
\end{gathered}
$$

and implies

$$
\frac{\left|w_{2}(\mathrm{j} \omega)\right|}{\sqrt{1+\gamma}} \leqslant \frac{\sigma_{i}\left(G_{\mathrm{a}}(\mathrm{j} \omega)\right)}{\sigma_{i}(G(\mathrm{j} \omega))} \leqslant \frac{\sqrt{1+\gamma}}{\left|w_{1}(\mathrm{j} \omega)\right|}
$$

for all $\omega$, and $i=1 \ldots p$.

\section{Proof}

We prove that (6) is equivalent to (9). The other equivalences are shown similarly. Notice that $\left\|w_{1} G^{-1} G_{\mathrm{a}}\right\|_{\infty} \leqslant \sqrt{1+\gamma}$ is equivalent to $\bar{\sigma}\left(G^{-1} G_{\mathrm{a}}\right) \leqslant \sqrt{1+\gamma} /\left|w_{1}\right|$, for all $\omega$. Since $\sigma_{i}(\cdot) \leqslant \bar{\sigma}(\cdot)$ the upper bound in (6) follows, and conversely the upper bound implies the first inequality of (9). Next notice that $\left\|w_{2} G_{\mathrm{a}}^{-1} G\right\|_{\infty} \leqslant \sqrt{1+\gamma}$ is equivalent to $\bar{\sigma}\left(G_{\mathrm{a}}^{-1} G\right) \leqslant \sqrt{1+\gamma} /\left|w_{2}\right|$ which is equivalent to $\underline{\sigma}\left(G^{-1} G_{\mathrm{a}}\right) \geqslant\left|w_{2}\right| / \sqrt{1+\gamma}$ for all $\omega$. Since $\sigma_{i}(\cdot) \geqslant \underline{\sigma}(\cdot)$ the lower bound in (6) follows, and conversely the lower bound implies the second inequality of (9).

The right hand inequality (10) follows from the right hand inequality of (6) if we observe the following generalization of the submultiplicative property of the singular values: $\sigma_{i}\left(G_{\mathrm{a}}\right) \leqslant$ $\sigma_{i}(G) \bar{\sigma}\left(G^{-1} G_{\mathrm{a}}\right)$, see [13, p. 423]. Similarly, the left hand inequality of (10) is derived by using the left inequality of (6) and the singular value property $\sigma_{i}(G) \underline{\sigma}\left(G^{-1} G_{\mathrm{a}}\right) \leqslant \sigma_{i}\left(G_{\mathrm{a}}\right)$.

The following corollary to Theorem 1 shows that if an approximation $G_{\text {a }}$ satisfies (6), then its gains $\sigma_{i}\left(G_{\mathrm{a}}(\mathrm{j} \omega)\right)$ are close to $\sigma_{i}(G(\mathrm{j} \omega))$. In particular, it shows that the difference between the 
logarithm of the singular values of $G$ and $G_{\mathrm{a}}$ is bounded. That is, the distance between the curves typically plotted for MIMO systems in Bode magnitude plots, is bounded.

\section{Corollary 2}

The inequalities (6) imply

$$
-\frac{1}{2} \log (1+\gamma)+\log \left|w_{2}(\mathrm{j} \omega)\right| \leqslant \log \sigma_{i}\left(G_{\mathrm{a}}(\mathrm{j} \omega)\right)-\log \sigma_{i}(G(\mathrm{j} \omega)) \leqslant \frac{1}{2} \log (1+\gamma)-\log \left|w_{1}(\mathrm{j} \omega)\right|
$$

for all $\omega$, and $i=1 \ldots p$.

Proof

Take the logarithm of (10).

Remark 2 (Constraints on $\gamma$ and $w_{1}, w_{2}$ )

From the previous inequalities, it should be clear why we require $\gamma \geqslant 0$ and $\left\|w_{1}\right\|_{\infty} \leqslant 1,\left\|w_{2}\right\|_{\infty} \leqslant 1$. If these conditions are violated, it is possible that a solution where $G_{\mathrm{a}}(\mathrm{j} \omega)=G(\mathrm{j} \omega)$, for some or all $\omega$, does not satisfy the constraints (6). This is obviously undesirable in an approximation problem.

The bounds (11) and the parameter $\gamma$ have a clear interpretation in a Bode diagram, see Figure 1. When $\gamma=0$ we are looking for $G_{\mathrm{a}}$ with magnitude function $\left|G_{\mathrm{a}}(\mathrm{j} \omega)\right|$ that satisfies the

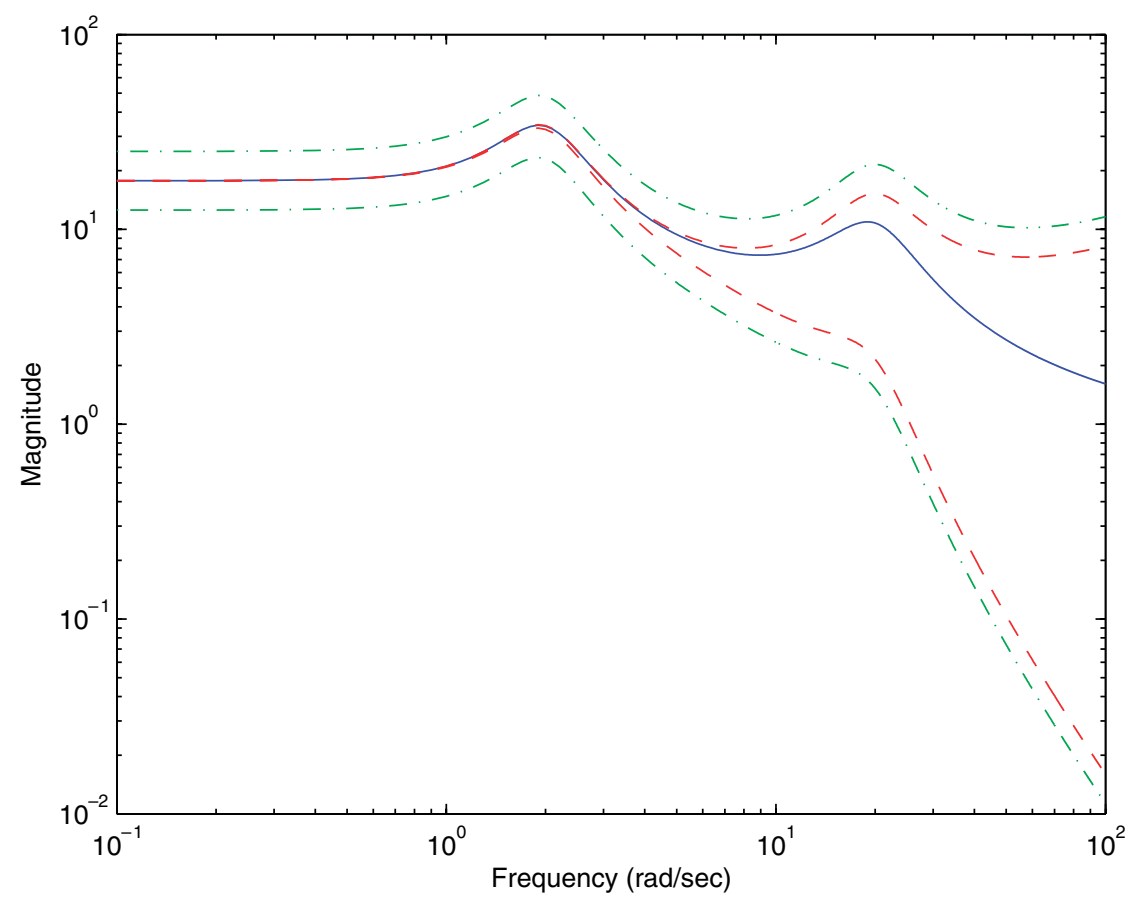

Figure 1. The interpretation of the weights $w_{1}, w_{2}$, and the constant $\gamma$. The full line represents the model $G$, the dashed lines represent the bounds with $\gamma=0$, and the dash-dotted lines the relaxed bounds with $\gamma=1$. The magnitude of the sought approximation $G_{\mathrm{a}}$ should be within the bounds with the smallest possible $\gamma$. 
tightest bounds in Figure 1. These bounds are given by $w_{1}$ and $w_{2}$. There may be such an approximation or not, depending on the weights $w_{1}, w_{2}$ and model $G$. When $\gamma>0$ the bounds are relaxed uniformly, as is seen in Figure 1. Hence, when $\gamma$ is minimized in (5), we are looking for an approximation that satisfies the tightest possible bounds. A similar situation holds in the MIMO case, but then for each singular value, as seen in (6) and (8)-(11). The approximation problem depicted in Figure 1 is solved in Section 4.

Remark 3 (Equivalence in SISO case)

When $G$ is SISO $(p=1)$ the inequalities (6) and (8)-(11) are stating the same thing, since $\sigma_{1}(G)=|G|$, and (11) is equivalent to (6). Hence, in the scalar case, if we solve (5)-(7) with $w_{1}=w_{2}=1$, a solution to the problem (1) is obtained.

Remark 4 (MIMO weights)

It is possible to use MIMO weights instead of scalar weights $w_{1}, w_{2}$. Then one should group the weights together with $G$, i.e. $G \rightarrow W_{0} G W_{i}$ and all the analysis in this section carries through. In general, it seems enough to use scalar weights, however. MIMO weights could be useful if one would like to have different approximation accuracy for different $\sigma_{i}$ in (6) and (8)-(10). Then one needs to carefully design the directional amplification of the weights.

\subsection{Parametrization and the semidefinite program}

In this section, we show how (5)-(7) is translated into a semidefinite program. We also show how the program may be solved when used for model reduction and system identification.

First we introduce the parametrization of $G_{\mathrm{a}}$. The parametrization is critical to be able to formulate the problem as a semidefinite program. Furthermore, it is also important that the parametrization contains all $G_{\mathrm{a}}$ that are interesting. It is shown in Theorem 3 that the following parametrization is a good choice and satisfies these conditions. Let $m$ be a positive integer (which will turn out to be related to the McMillan degree of the approximant $G_{\mathrm{a}}$ ) and define an even scalar polynomial

$$
a_{m}\left(\omega^{2}\right):=\omega^{2 m}+a_{2 m-2} \omega^{2 m-2}+\cdots+a_{2} \omega^{2}+a_{0}
$$

and a hermitian polynomial matrix

$$
B_{m}(\omega):=\left(\begin{array}{ccc}
b_{2 m}^{11} \omega^{2 m}+b_{2 m-2}^{11} \omega^{2 m-2}+\cdots+b_{0}^{11} & \cdots & {\left[\left(b_{2 m}^{p 1} \omega^{2 m}+b_{2 m-2}^{p 1} \omega^{2 m-2}+\cdots+b_{0}^{p 1}\right)\right.} \\
\vdots & & \left.-\mathrm{j} \omega\left(b_{2 m-1}^{p 1} \omega^{2 m-2}+\cdots+b_{1}^{p 1}\right)\right] \\
{\left[\left(b_{2 m}^{p 1} \omega^{2 m}+b_{2 m-2}^{p 1} \omega^{2 m-2}+\cdots+b_{0}^{p 1}\right)\right.} & \ddots & \vdots \\
\left.+\mathrm{j} \omega\left(b_{2 m-1}^{p 1} \omega^{2 m-2}+\cdots+b_{1}^{p 1}\right)\right] & \cdots & b_{2 m}^{p p} \omega^{2 m}+b_{2 m-2}^{p p} \omega^{2 m-2}+\cdots+b_{0}^{p p}
\end{array}\right)
$$

where $\left\{a_{i}\right\}$ and $\left\{b_{i}^{\mathrm{j} k}\right\}, 0 \leqslant i \leqslant 2 m, 1 \leqslant k \leqslant \mathrm{j} \leqslant p$, are the decision variables. Notice that $B_{m}(\omega)=B_{m}(-\omega)^{\mathrm{T}}=B_{m}(\omega)^{*} \in \mathbb{C}^{p \times p}$. The polynomial $a_{m}\left(\omega^{2}\right)$ has $m$ decision variables, and $B_{m}(\omega)$ has $(p / 2)(2 p m+p+1)$ decision variables. 
Theorem 3

Assume that $G, w_{1}, w_{2}$ satisfy Assumption 1, and that the integer $m$ and $\gamma \geqslant 0$ are fixed. If there is a $G_{\mathrm{a}} \in R_{m} H_{\infty}$ that satisfies (6), then there exist $a_{m}, B_{m}$ that satisfy

$$
\left|w_{1}(\mathrm{j} \omega)\right|^{2} B_{m}(\omega)-(1+\gamma) a_{m}\left(\omega^{2}\right)\left(G G^{*}\right)(\mathrm{j} \omega) \leqslant 0
$$

and

$$
\left|w_{2}(\mathrm{j} \omega)\right|^{2} a_{m}\left(\omega^{2}\right)\left(G G^{*}\right)(\mathrm{j} \omega)-(1+\gamma) B_{m}(\omega) \leqslant 0
$$

and

$$
a_{m}\left(\omega^{2}\right)>0
$$

for all $\omega$. Conversely, if there are $a_{m}, B_{m}$ that satisfy (12)-(14) for all $\omega$, then there is a $G_{\mathrm{a}} \in$ $R_{m p} H_{\infty}$ that satisfies (6) and $G_{\mathrm{a}}^{-1} \in R_{m p} H_{\infty}$.

Proof

Assume that $G_{\mathrm{a}} \in R_{m} H_{\infty}$ and that it satisfies (6). According to Theorem 1, $G_{\mathrm{a}}$ then satisfies (9). The inequalities in (9) imply

$$
\left|w_{1}(\mathrm{j} \omega)\right|^{2}\left(G_{\mathrm{a}} G_{\mathrm{a}}^{*}\right)(\mathrm{j} \omega)-(1+\gamma)\left(G G^{*}\right)(\mathrm{j} \omega) \leqslant 0
$$

and

$$
\left|w_{2}(\mathrm{j} \omega)\right|^{2}\left(G G^{*}\right)(\mathrm{j} \omega)-(1+\gamma)\left(G_{\mathrm{a}} G_{\mathrm{a}}^{*}\right)(\mathrm{j} \omega) \leqslant 0
$$

for all $\omega$. We have that $G_{\mathrm{a}}(\mathrm{j} \omega)=C(\mathrm{j} \omega I-A)^{-1} B+D$ where $A \in \mathbb{R}^{m \times m}, B \in \mathbb{R}^{m \times p}, C \in \mathbb{R}^{p \times m}$, and $D \in \mathbb{R}^{p \times p}$. Notice that $\left(G_{\mathrm{a}} G_{\mathrm{a}}^{*}\right)(\mathrm{j} \omega)$ can be written as $B(\omega) / a(\omega)$ where

$$
\begin{gathered}
a(\omega)=\operatorname{det}(\mathrm{j} \omega I-A) \operatorname{det}(-\mathrm{j} \omega I-A) \\
B(\omega)=(C \operatorname{adj}(\mathrm{j} \omega I-A) B+D \operatorname{det}(\mathrm{j} \omega I-A)) \\
\times\left(B^{\mathrm{T}} \operatorname{adj}(-\mathrm{j} \omega I-A)^{\mathrm{T}} C^{\mathrm{T}}+D^{\mathrm{T}} \operatorname{det}(-\mathrm{j} \omega I-A)\right)
\end{gathered}
$$

and both $a(\omega)$ and $B(\omega)$ contain polynomials up to order $2 m$. Since $a(\omega)=a(-\omega)$ and $B(\omega)=B(\omega)^{*}=B(-\omega)^{\mathrm{T}}$ we have $a(\omega)=a_{m}\left(\omega^{2}\right)$ and $B(\omega)=B_{m}(\omega)$ for some $\left\{a_{i}\right\}$ and $\left\{b_{i}^{\mathrm{j} k}\right\}$. Furthermore, since $G_{\mathrm{a}} \in R_{m} H_{\infty}$ it can always be realized with an $A$ that has no eigenvalues on the imaginary axis, and we have $a(\omega)=a_{m}\left(\omega^{2}\right)>0$ for all $\omega$, which gives (14). Finally, if we multiply (15)-(16) with $a_{m}\left(\omega^{2}\right)$, we obtain (12)-(13). This concludes the first part of the proof. Next, we prove the converse result.

Define

$$
\Phi(s):=\frac{1}{a_{m}\left(\omega^{2}\right)} B_{m}(\omega) \quad \text { using } \omega^{2 n} \leftarrow(-1)^{n} s^{2 n}, \quad \mathrm{j} \omega^{2 n-1} \leftarrow(-1)^{n+1} s^{2 n-1}, \quad n=1 \ldots m
$$

Divide (12)-(13) with $a_{m}\left(\omega^{2}\right)$ and notice that $\Phi(\mathrm{j} \omega)=B_{m}(\omega) / a_{m}\left(\omega^{2}\right)$ satisfies

$$
\Phi(\mathrm{j} \omega) \leqslant \frac{1+\gamma}{\left|w_{1}(\mathrm{j} \omega)\right|^{2}}\left(G G^{*}\right)(\mathrm{j} \omega), \quad \Phi(\mathrm{j} \omega)^{-1} \leqslant \frac{1+\gamma}{\left|w_{2}(\mathrm{j} \omega)\right|^{2}}\left(G G^{*}\right)(\mathrm{j} \omega)^{-1}
$$

and $\Phi, \Phi^{-1} \in L_{\infty}$ because of our assumption that $G, G^{-1}, w_{1}^{-1}, w_{2}^{-1} \in L_{\infty}$. Notice that $\Phi^{-1}$ is well defined since $B_{m}(\omega)$ is invertible for all $\omega$. This follows from (13)-(14) and the assumptions 
that $G, w_{2}$ have no zeroes on the imaginary axis $\left(G^{-1}, w_{2}^{-1} \in L_{\infty}\right)$, since

$$
0<\frac{1}{(1+\gamma)}\left|w_{2}(\mathrm{j} \omega)\right|^{2} a_{m}\left(\omega^{2}\right)\left(G G^{*}\right)(\mathrm{j} \omega) \leqslant B_{m}(\omega)
$$

for all $\omega$. Finally notice that $\Phi(s)$ is parahermitian, i.e. $\Phi(s)=\Phi(-s)^{\mathrm{T}}, s \in \mathbb{C}$. Using standard results on spectral factorizations, see, for example, [14], it follows that $\Phi(s)$ has a spectral factor $G_{\mathrm{a}}, G_{\mathrm{a}}^{-1} \in R H_{\infty}$ such that $\Phi(s)=G_{\mathrm{a}}(s) G_{\mathrm{a}}(-s)^{\mathrm{T}}$. Also notice that $\Phi(s)$ has a realization of McMillan degree less or equal to $2 m p$. Hence, $G_{\mathrm{a}} \in R_{m p} H_{\infty}$. Using the spectral factorization we see that (12)-(13) gives

$$
\left(w_{1} G^{-1} G_{\mathrm{a}}\right)\left(w_{1} G^{-1} G_{\mathrm{a}}\right)^{*}(\mathrm{j} \omega) \leqslant 1+\gamma
$$

and

$$
\left(w_{2} G_{\mathrm{a}}^{-1} G\right)\left(w_{2} G_{\mathrm{a}}^{-1} G\right)^{*}(\mathrm{j} \omega) \leqslant 1+\gamma
$$

for all $\omega$. This is equivalent to (9) and (6), and concludes the proof.

Remark 5 (Equivalence in SISO case)

Theorem 3 is stronger in the SISO case when $p=1$. Then there is an approximation $G_{\text {a }}$ of McMillan degree $m$ that satisfies (6) if and only if (12)-(14) holds. In the MIMO case, a $G_{\text {a }}$ of McMillan degree $m$ can only result when the decision variables in $a_{m}\left(\omega^{2}\right)$ and $B_{m}(\omega)$ lie in a proper algebraic variety, corresponding to requiring the residue matrix of $\Phi(s)$ at each zero of the denominator taking rank 1 .

Remark 6 (Strictly proper $G$ and zeroes on imaginary axis)

We have made the assumptions in Theorem 3 that $G^{-1} \in R L_{\infty}$. This means that $G$ is biproper and that it has no zeroes on the imaginary axis. This assumption can be relaxed if there is an integer $n$ such that $\lim _{s \rightarrow \infty} \operatorname{det}\left((s+1)^{n} G(s)\right) \neq 0$. Then one can apply the method in the proof of Theorem 3 using $\tilde{G}(\mathrm{j} \omega)=(\mathrm{j} \omega+1)^{n} G(\mathrm{j} \omega)$ and $\tilde{B}_{m}(\omega)=\left(\omega^{2}+1\right)^{n} B_{m-n}(\omega)$ instead of $G$ and $B_{m}$. We then obtain $\tilde{\Phi}(\mathrm{j} \omega) \geqslant 0, \tilde{\Phi}(s)=\tilde{\Phi}(-s)^{\mathrm{T}}$ and $\operatorname{det} \tilde{\Phi}(\infty) \neq 0$. This is enough for existence of a spectral factor $\tilde{G}_{\mathrm{a}}, \tilde{G}_{\mathrm{a}}^{-1} \in R_{m p} H_{\infty}$ (with $n$ zeroes at $s=-1$ ), see [15]. Finally, we can construct a minimum phase $G_{\mathrm{a}} \in R_{m p} H_{\infty}$ from a minimal realization of $\tilde{G}_{\mathrm{a}}(s) /(s+1)^{n}$.

For fixed $\gamma,(12)-(14)$ is a convex feasibility problem in the decision variables $\left\{a_{i}\right\}$ and $\left\{b_{i}^{\mathrm{j} k}\right\}$. This is because $a_{m}$ and $B_{m}$ are linear in the decision variables. Using Theorem 3 in the SISO case, we can thus replace (6)-(7) with the convex feasibility problem (12)-(14). This implies that the minimization problem (5)-(7) is quasiconvex [12, p. 145], and that it can be solved by means of a bisection algorithm.

In the MIMO case, we can still replace (6)-(7) with (12)-(14), and we again obtain a quasiconvex minimization problem. However, we can now only guarantee that the solutions belong to $R_{m p} H_{\infty}$. More precisely, we can obtain those approximations $G_{\mathrm{a}} \in R_{m p} H_{\infty}$ that can be written as $G_{\mathrm{a}} G_{\mathrm{a}}^{*}=B_{m} / a_{m}$. This includes all $G_{\mathrm{a}} \in R_{m} H_{\infty}$.

The inequalities (12)-(14) consist of an infinite number of constraints because of the dependence on $\omega$. If a state-space realization of $G(\mathrm{j} \omega)$ is known, such as in a typical model reduction situation, this dependence can be removed using the Kalman-Yakubovich-Popov (KYP) lemma. The KYP lemma was derived in [16, 17], and its original use is exposed in detail by Yakubovich et al. [18]. Here we will use the formulation given by Rantzer [19]. As an 
alternative to the KYP lemma, a sum of squares decomposition [20] could be used. If $G(\mathrm{j} \omega)$ is only known on a grid $\left\{\omega_{k}\right\}$, such as in the typical system identification situation, Theorem 3 can still be used with a slight modification. These issues are discussed next.

2.1.1. Magnitude model reduction and the KYP lemma. Here we assume that the model $G$ we shall approximate and the weights $w_{1}, w_{2}$ are given in state-space form. The easiest condition out of (12)-(14) to translate into a semidefinite program is the third inequality (14). Define

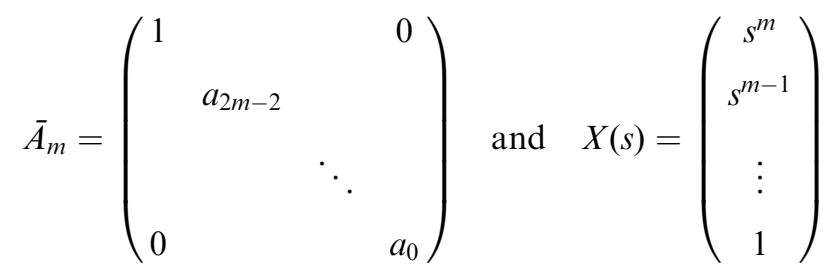

where $\left\{a_{i}\right\}$ are the decision variables. Then (14) is given by

$$
a_{m}\left(\omega^{2}\right)=X(\mathrm{j} \omega)^{*} \bar{A}_{m} X(\mathrm{j} \omega)>0
$$

for all $\omega$. If we divide (18) with $\alpha(\mathrm{j} \omega)^{*} \alpha(\mathrm{j} \omega)$, where $\alpha(s)$ is any $m$ th order polynomial without roots on the imaginary axis, (18) can equivalently be written as

$$
\left[\begin{array}{c}
\left(\mathrm{j} \omega I-A_{3}\right)^{-1} B_{3} \\
1
\end{array}\right]^{*}\left[\begin{array}{ll}
C_{3} & D_{3}
\end{array}\right]^{*} \bar{A}_{m}\left[\begin{array}{ll}
C_{3} & D_{3}
\end{array}\right]\left[\begin{array}{c}
\left(\mathrm{j} \omega I-A_{3}\right)^{-1} B_{3} \\
1
\end{array}\right]>0
$$

for all $\omega$. Here $\left(A_{3}, B_{3}, C_{3}, D_{3}\right)$ is a minimal realization of McMillan degree $m$ such that

$$
X(s) / \alpha(s)=C_{3}\left(s I-A_{3}\right)^{-1} B_{3}+D_{3}
$$

If we use the KYP lemma as formulated by Rantzer [19], inequality (19) is equivalent to the existence of a symmetric matrix $P_{3}=P_{3}^{\mathrm{T}} \in \mathbb{R}^{m \times m}$ such that

$$
\left[\begin{array}{cc}
A_{3}^{\mathrm{T}} P_{3}+P_{3} A_{3} & P_{3} B_{3} \\
B_{3}^{\mathrm{T}} P_{3} & 0
\end{array}\right]-\left[\begin{array}{ll}
C_{3} & D_{3}
\end{array}\right]^{*} \bar{A}_{m}\left[\begin{array}{ll}
C_{3} & D_{3}
\end{array}\right]<0
$$

Inequality (20) is an LMI in the unknowns $\bar{A}_{m} \in \mathbb{R}^{(m+1) \times(m+1)}$ and $P_{3} \in \mathbb{R}^{m \times m}$ and is readily solved using standard tools in MaTLAB such as SeDuMi [21] together with YALMIP [22].

The conditions (12)-(13) can also be turned into LMIs for fixed $\gamma$. Define

$$
\bar{B}_{m}=\left[\begin{array}{ccc}
B_{11} & \ldots & B_{1 p} \\
\vdots & \ddots & \vdots \\
B_{p 1} & \ldots & B_{p p}
\end{array}\right], \quad B_{i i}=\left(\begin{array}{cccc}
b_{2 m}^{i i} & & & 0 \\
& b_{2 m-2}^{i i} & & \\
& & \ddots & \\
0 & & & b_{0}^{i i}
\end{array}\right), \quad B_{i j} \stackrel{(>>j)}{=}\left(\begin{array}{cccc}
b_{2 m}^{i j} & & & 0 \\
b_{2 m-1}^{i j} & b_{2 m-2}^{i j} & & \\
& \ddots & \ddots & \\
& & b_{1}^{i j} & b_{0}^{i j}
\end{array}\right)
$$

and $B_{i j}=B_{j i}^{\mathrm{T}}$ when $i<j$, where $\left\{b_{i}^{\mathrm{j} k}\right\}$ are the decision variables. Then notice that

$$
\begin{gathered}
B_{m}(\omega)=\left(I_{p} \otimes X(\mathrm{j} \omega)\right)^{*} \bar{B}_{m}\left(I_{p} \otimes X(\mathrm{j} \omega)\right) \\
a_{m}\left(\omega^{2}\right)\left(G G^{*}\right)(\mathrm{j} \omega)=\left(G^{\sim} \otimes X(\mathrm{j} \omega)\right)^{*}\left(I_{p} \otimes \bar{A}_{m}\right)\left(G^{\sim} \otimes X(\mathrm{j} \omega)\right)
\end{gathered}
$$


where $\otimes$ is the Kronecker (direct) product. Hence, the inequality (12) is equivalent to

$$
\left[\begin{array}{c}
I_{p} \otimes\left(w_{1} X\right)(\mathrm{j} \omega) \\
\left(G^{\sim} \otimes X\right)(\mathrm{j} \omega)
\end{array}\right]^{*}\left[\begin{array}{cc}
\bar{B}_{m} & 0 \\
0 & -(1+\gamma)\left(I_{p} \otimes \bar{A}_{m}\right)
\end{array}\right]\left[\begin{array}{c}
I_{p} \otimes\left(w_{1} X\right)(\mathrm{j} \omega) \\
\left(G^{\sim} \otimes X\right)(\mathrm{j} \omega)
\end{array}\right] \leqslant 0
$$

for all $\omega$. Next introduce a minimal realization $\left(A_{1}, B_{1}, C_{1}, D_{1}\right)$ such that

$$
\frac{1}{\alpha(s)}\left[\begin{array}{c}
I_{p} \otimes\left(w_{1} X\right)(s) \\
\left(G^{\sim} \otimes X\right)(s)
\end{array}\right]=C_{1}\left(s I-A_{1}\right)^{-1} B_{1}+D_{1}
$$

Let us denote the McMillan degree of $\left(A_{1}, B_{1}, C_{1}, D_{1}\right)$ by $n_{1}$. The degree $n_{1}$ depends on $p$, $m$, and the degrees of $G$ and $w_{1}$. Using the KYP lemma, (21) is equivalent to the existence of a symmetric $P_{1} \in \mathbb{R}^{n_{1} \times n_{1}}$ such that the LMI

$$
\left[\begin{array}{cc}
A_{1}^{\mathrm{T}} P_{1}+P_{1} A_{1} & P_{1} B_{1} \\
B_{1}^{\mathrm{T}} P_{1} & 0
\end{array}\right]+\left[\begin{array}{cc}
C_{1} & D_{1}
\end{array}\right]^{*}\left[\begin{array}{cc}
\bar{B}_{m} & 0 \\
0 & -(1+\gamma)\left(I_{p} \otimes \bar{A}_{m}\right)
\end{array}\right]\left[\begin{array}{cc}
C_{1} & D_{1}
\end{array}\right] \leqslant 0
$$

holds. The inequality (13) is in a similar way written as

$$
\left[\begin{array}{c}
I_{p} \otimes X(\mathrm{j} \omega) \\
\left(w_{2} G^{\sim} \otimes X\right)(\mathrm{j} \omega)
\end{array}\right]^{*}\left[\begin{array}{cc}
-(1+\gamma) \bar{B}_{m} & 0 \\
0 & I_{p} \otimes \bar{A}_{m}
\end{array}\right]\left[\begin{array}{c}
I_{p} \otimes X(\mathrm{j} \omega) \\
\left(w_{2} G^{\sim} \otimes X\right)(\mathrm{j} \omega)
\end{array}\right] \leqslant 0
$$

which again is equivalent to the existence of a symmetric $P_{2} \in \mathbb{R}^{n_{2} \times n_{2}}$ such that

$$
\left[\begin{array}{cc}
A_{2}^{\mathrm{T}} P_{2}+P_{2} A_{2} & P_{2} B_{2} \\
B_{2}^{\mathrm{T}} P_{2} & 0
\end{array}\right]+\left[\begin{array}{ll}
C_{2} & D_{2}
\end{array}\right]^{*}\left[\begin{array}{cc}
-(1+\gamma) \bar{B}_{m} & 0 \\
0 & I_{p} \otimes \bar{A}_{m}
\end{array}\right]\left[\begin{array}{ll}
C_{2} & D_{2}
\end{array}\right] \leqslant 0
$$

if we choose a minimal realization

$$
\frac{1}{\alpha(s)}\left[\begin{array}{c}
I_{p} \otimes X(s) \\
\left(w_{2} G^{\sim} \otimes X\right)(s)
\end{array}\right]=C_{2}\left(s I-A_{2}\right)^{-1} B_{2}+D_{2}
$$

of degree $n_{2}$.

The magnitude model reduction problem (5)-(7) (the modified problem in the MIMO case) can now be solved with a bisection algorithm.

Algorithm 1 (Magnitude model reduction)

Input: transfer functions $G(s), w_{1}(s), w_{2}(s)$ that satisfy Assumption 1, an integer $m$, and tolerance $\varepsilon_{\mathrm{tol}}>0$. Output: $G_{\mathrm{a}}, G_{\mathrm{a}}^{-1} \in R_{m p} H_{\infty}$ that solves the (modified) problem (5)-(7) with accuracy $\varepsilon_{\mathrm{tol}}$.

1. If (20), (22) and (23) are feasible with $\gamma=\gamma_{u}=0$, goto 6 .

2. Put $\gamma_{l}=0$ and $\gamma_{u}=\left(\max \left\{\left\|w_{1} G^{-1}\right\|_{\infty},\left\|w_{2} G\right\|_{\infty}\right\}\right)^{2}-1$.

3. Put $\gamma_{t}=\left(\gamma_{u}+\gamma_{l}\right) / 2$.

4. If (20), (22) and (23) are feasible with $\gamma=\gamma_{t}$, then $\gamma_{u}=\gamma_{t}$, else $\gamma_{l}=\gamma_{t}$.

5. If $\gamma_{u}-\gamma_{l}>\varepsilon_{\text {tol }}$ goto 3 .

6. From the feasible solution associated with $\gamma_{u}$, compute a spectral factorization of $B_{m}(\omega) / a_{m}\left(\omega^{2}\right)$, such that $G_{\mathrm{a}}, G_{\mathrm{a}}^{-1} \in R_{m p} H_{\infty}$ and

$$
B_{m}(\omega) / a_{m}\left(\omega^{2}\right)=G_{\mathrm{a}}(\mathrm{j} \omega) G_{\mathrm{a}}(\mathrm{j} \omega)^{*}
$$


Remark 7 (Existence and McMillan degree of solutions)

Notice that Theorem 3 guarantees that if there is a $G_{\mathrm{a}} \in R_{m} H_{\infty}$ that satisfies (6), then there are solutions $P_{1}, P_{2}, P_{3}, \bar{A}_{m}, \bar{B}_{m}$ subject to the LMIs (20), (22) and (23). But for the spectral factor of a solution $B_{m}(\omega) / a_{m}\left(\omega^{2}\right)$, we can only guarantee that it has a McMillan degree less or equal to $m p$. This apparent contradiction is due to the fact that the LMIs do generally not have a unique solution, and we do not know how to enforce a condition that gives the solution with smallest McMillan degree. This is perhaps easily understood by means of an example. Assume that there exists a good approximation

$$
G_{\mathrm{a}}^{(1)}(s)=\left(\begin{array}{cc}
\frac{s+1}{s+2} & 0 \\
0 & \frac{s+3}{s+4}
\end{array}\right)
$$

that satisfies (6) for some $G$ and very small $\gamma$ without weights. If we solve (20), (22) and (23) using the same $G$ and $\gamma$ it is, however, more likely that we obtain something similar to

$$
G_{\mathrm{a}}^{(2)}(s)=\left(\begin{array}{cc}
\frac{(s+1)\left(s+4+\varepsilon_{1}\right)}{(s+2)(s+4)} & 0 \\
0 & \frac{(s+3)\left(s+2+\varepsilon_{2}\right)}{(s+2)(s+4)}
\end{array}\right)
$$

for some small $\varepsilon_{1}, \varepsilon_{2}$. The approximation $G_{\mathrm{a}}^{(1)}$ has McMillan degree $m=2$ whereas $G_{\mathrm{a}}^{(2)}$ has McMillan degree $m p=4$. Because of this, it is recommended that one applies balanced truncation or Hankel norm approximation to the approximations $G_{\mathrm{a}} \in R_{m p} H_{\infty}$. This should not be a large computational burden since $G_{\mathrm{a}}$ should already be of relatively low order. Notice that all this only applies in the MIMO case. In the SISO case, we will directly find a solution of McMillan degree $m$, if it exists.

2.1.2. Magnitude system identification and finite number of data points. In the system identification case, we only assume knowledge of $G(\mathrm{j} \omega)$ on a grid $\left\{\omega_{k}\right\}$. Then it is of course not meaningful to use the constraint (9). What we can do instead is to find approximations $G_{\text {a }}$ such that

$$
\max _{k} \bar{\sigma}\left(w_{1} G^{-1} G_{\mathrm{a}}\right)\left(\mathrm{j} \omega_{k}\right) \leqslant \sqrt{1+\gamma}
$$

and

$$
\max _{k} \bar{\sigma}\left(w_{2} G_{\mathrm{a}}^{-1} G\right)\left(\mathrm{j} \omega_{k}\right) \leqslant \sqrt{1+\gamma}
$$

(which correspond to (9)) using a slight modification of Theorem 1. The inequalities (12)-(13) are easily enforced on $\left\{\omega_{k}\right\}$ since they are already LMIs in the variables $\left\{a_{i}\right\}$ and $\left\{b_{i}^{\mathrm{j} k}\right\}$ for fixed $\omega$. The KYP lemma is not needed for them since the number of constraints is finite. However, to guarantee that a spectral factorization of $B_{m}(\omega) / a_{m}\left(\omega^{2}\right)$ exists, LMI (20) must still be used, and furthermore, we also need to introduce a second LMI, analog to (20), to guarantee that $B_{m}(\omega)>0$ for all $\omega$. Using the previous definitions, we have

$$
B_{m}(\omega)=\left(I_{p} \otimes X(\mathrm{j} \omega)\right)^{*} \bar{B}_{m}\left(I_{p} \otimes X(\mathrm{j} \omega)\right)>0
$$


for all $\omega$. The KYP lemma says this is equivalent to the existence of a symmetric $P_{4} \in \mathbb{R}^{n_{4} \times n_{4}}$ such that

$$
\left[\begin{array}{cc}
A_{4}^{\mathrm{T}} P_{4}+P_{4} A_{4} & P_{4} B_{4} \\
B_{4}^{\mathrm{T}} P_{4} & 0
\end{array}\right]-\left[\begin{array}{ll}
C_{4} & D_{4}
\end{array}\right]^{*} \bar{B}_{m}\left[\begin{array}{ll}
C_{4} & D_{4}
\end{array}\right]<0
$$

if we choose a minimal realization

$$
\frac{1}{\alpha(s)}\left[I_{p} \otimes X(s)\right]=C_{4}\left(s I-A_{4}\right)^{-1} B_{4}+D_{4}
$$

of degree $n_{4}$. The LMI (24) was not necessary in the model reduction case since $B_{m}(\omega)>0$ followed automatically. See (17) in the proof of Theorem 3.

\subsection{Relative $H_{\infty}$ error bounds}

In this section, we show that not only does (6) give simple bounds on the singular values, as shown in Theorem 1 and Corollary 2, but we can also obtain a priori bounds on the relative $H_{\infty}$ norm error. As is discussed in [4], relative error bounds on plant models are more useful than additive bounds if the plant is used in closed-loop systems.

\section{Theorem 4}

Assume that $G, G_{\mathrm{a}} \in R L_{\infty}$. The inequalities

$$
1 / \sqrt{1+\gamma} \leqslant \sigma_{i}\left(\left(G^{-1} G_{\mathrm{a}}\right)(\mathrm{j} \omega)\right) \leqslant \sqrt{1+\gamma}
$$

for all $\omega$ and $i=1 \ldots p$, are equivalent to

$$
\left\|\left(G^{-1} G_{\mathrm{a}}\right)\left(G^{-1} G_{\mathrm{a}}\right)^{*}-I\right\|_{\infty} \leqslant \gamma
$$

and

$$
\left\|\left(G_{\mathrm{a}}^{-1} G\right)\left(G_{\mathrm{a}}^{-1} G\right)^{*}-I\right\|_{\infty} \leqslant \gamma
$$

If furthermore $\left(G^{-1} G_{\mathrm{a}}\right),\left(G_{\mathrm{a}}^{-1} G\right) \in R_{n} H_{\infty}$ and $\left(G^{-1} G_{\mathrm{a}}\right)(\infty)=I$, then (25) implies

$$
\left\|G^{-1}\left(G-G_{\mathrm{a}}\right)\right\|_{\infty} \leqslant 2 n \gamma \sqrt{1+\gamma}
$$

and

$$
\left\|G_{\mathrm{a}}^{-1}\left(G-G_{\mathrm{a}}\right)\right\|_{\infty} \leqslant 2 n \gamma \sqrt{1+\gamma}
$$

Proof

Notice that $\left\|\left(G^{-1} G_{\mathrm{a}}\right)\left(G^{-1} G_{\mathrm{a}}\right)^{*}-I\right\|_{\infty} \leqslant \gamma$ is equivalent to (for all $\omega$ )

$$
\left(G_{\mathrm{a}} G_{\mathrm{a}}^{*}\right)(\mathrm{j} \omega)-(1+\gamma)\left(G G^{*}\right)(\mathrm{j} \omega) \leqslant 0
$$

and

$$
(1-\gamma)\left(G G^{*}\right)(\mathrm{j} \omega)-\left(G_{\mathrm{a}} G_{\mathrm{a}}^{*}\right)(\mathrm{j} \omega) \leqslant 0
$$

and that $\left\|\left(G_{\mathrm{a}}^{-1} G\right)\left(G_{\mathrm{a}}^{-1} G\right)^{*}-I\right\|_{\infty} \leqslant \gamma$ is equivalent to (for all $\omega$ )

$$
\left(G G^{*}\right)(\mathrm{j} \omega)-(1+\gamma)\left(G_{\mathrm{a}} G_{\mathrm{a}}^{*}\right)(\mathrm{j} \omega) \leqslant 0
$$


and

$$
(1-\gamma)\left(G_{\mathrm{a}} G_{\mathrm{a}}^{*}\right)(\mathrm{j} \omega)-\left(G G^{*}\right)(\mathrm{j} \omega) \leqslant 0
$$

If $0 \leqslant \gamma<1$, (28) is equivalent to $\left\|G^{-1} G_{\mathrm{a}}\right\|_{\infty} \leqslant \sqrt{1+\gamma}$ and $\left\|G_{\mathrm{a}}^{-1} G\right\|_{\infty} \leqslant 1 / \sqrt{1-\gamma}$. Under the same assumption, (29) is equivalent to $\left\|G_{\mathrm{a}}^{-1} G\right\|_{\infty} \leqslant \sqrt{1+\gamma}$ and $\left\|G^{-1} G_{\mathrm{a}}\right\|_{\infty} \leqslant 1 / \sqrt{1-\gamma}$. Since $\sqrt{1+\gamma}$ $\leqslant 1 / \sqrt{1-\gamma}$ for $0 \leqslant \gamma<1$, the equivalence with (25) asserted in the theorem statement follows for these $\gamma$, if we use Theorem 1. For $\gamma \geqslant 1$, the second inequalities of (28) and (29) are trivially satisfied, and the equivalence follows again.

The inequalities (27) can be shown using a similar technique as in [10, Theorem 6.1]. We only show the first inequality here. We have

$$
\begin{aligned}
\left\|G^{-1} G_{\mathrm{a}}-\left(G^{-1} G_{\mathrm{a}}\right)^{-*}\right\|_{\infty} & \left.=\|\left(\left(G^{-1} G_{\mathrm{a}}\right)\left(G^{-1} G_{\mathrm{a}}\right)^{*}-I\right)\left(G^{-1} G_{\mathrm{a}}\right)^{-*}\right) \|_{\infty} \\
& \leqslant \gamma\left\|\left(G^{-1} G_{\mathrm{a}}\right)^{-*}\right\|_{\infty} \leqslant \gamma \sqrt{1+\gamma}
\end{aligned}
$$

using (9) and (26). Next we use the Hankel singular values defined in, for example, [14]. Let $\sigma_{1}^{\mathrm{H}}\left(G^{-1} G_{\mathrm{a}}\right), \ldots, \sigma_{n}^{\mathrm{H}}\left(G^{-1} G_{\mathrm{a}}\right)$ denote the $n$ Hankel singular values of $G^{-1} G_{\mathrm{a}} \in R_{n} H_{\infty}$ in decreasing order. By Nehari's theorem (see, for example, [14, Theorem 8.20]) we then have

$$
\sigma_{1}^{\mathrm{H}}\left(G^{-1} G_{\mathrm{a}}\right) \leqslant\left\|G^{-1} G_{\mathrm{a}}-\left(G^{-1} G_{\mathrm{a}}\right)^{-*}\right\|_{\infty}
$$

since $\left(G^{-1} G_{\mathrm{a}}\right)^{-*} \in R H_{\infty}^{-}$. Hence, $\sigma_{1}^{\mathrm{H}}\left(G^{-1} G_{\mathrm{a}}\right) \leqslant \gamma \sqrt{1+\gamma}$. The bound [14, Corollary 7.4] now gives

$$
\left\|G^{-1} G_{\mathrm{a}}-\left(G^{-1} G_{\mathrm{a}}\right)(\infty)\right\|_{\infty} \leqslant 2 \sum_{i=1}^{n} \sigma_{i}^{\mathrm{H}}\left(G^{-1} G_{\mathrm{a}}\right) \leqslant 2 n \sigma_{1}^{\mathrm{H}}\left(G^{-1} G_{\mathrm{a}}\right) \leqslant 2 n \gamma \sqrt{1+\gamma}
$$

Using the fact that $\left(G^{-1} G_{\mathrm{a}}\right)(\infty)=I$ the first inequality in (27) follows. The second inequality is shown similarly.

The second part of Theorem 4 shows that if $G^{-1} G_{\mathrm{a}}$ is rational, biproper, stable, and minimum phase, $G_{\text {a }}$ can be proven to be a fine approximation of $G$ even if we have only matched magnitude data in (25) and (26). This is because the phase can be reconstructed from the magnitude with the Hilbert transform, and the error propagation can be bounded for rational functions. Notice in particular that the bound depends on the model order $n$. This is unfortunate but necessary since the Hilbert transform is not a bounded mapping for infinite-dimensional systems. For more details and comments about the tightness of bounds of this sort, see [10].

\section{PHASE APPROXIMATION}

In this section, we solve the phase approximation problem. We will approximate a transfer function $G$ with another transfer function $G_{\text {a }}$ (of smaller degree) such that the phases $\arg G(\mathrm{j} \omega)$ and $\arg G_{\mathrm{a}}(\mathrm{j} \omega)$ are close. The solution is similar to the magnitude case, but the parametrization is more complicated here. In particular, we only consider SISO problems. The reason is the splintering of the definition of phase of a MIMO system. For example, the phase of a MIMO system can be defined via phase functions, see [9, 10], or via the eigenvalues of $G(\mathrm{j} \omega)$, see [8]. How to construct the parametrization in Section 3.1 for the MIMO case is less obvious. 
The approximations that we obtain here will belong to a subset $Q_{m}$ of the rational transfer functions. The set is defined as

$$
Q_{m}=\left\{G(s): G(s)=\frac{f(s)}{g(s)}, f, g \text { are Hurwitz polynomials, } \operatorname{deg} f(s)+\operatorname{deg} g(s)=m\right\}
$$

If $G_{\mathrm{a}} \in Q_{m}$, it is minimum phase since the zeroes are in the left complex half plane. Since we put restrictions on $\arg G_{\mathrm{a}}(\mathrm{j} \omega)$ in this section, we can often bound the McMillan degree of the approximations $G_{\mathrm{a}}$, see Lemma 6 .

Before stating the optimization problem, we need some assumptions on the model $G$ and the weights $w_{1}, w_{2}$.

\section{Assumption 2}

$G, w_{1}, w_{2} \in R L_{\infty}$ and are scalar, $G, w_{1}, w_{2}$ have no zeroes on the imaginary axis, $\arg w_{1}(\mathrm{j} \omega) \leqslant$ $0, \arg w_{2}(\mathrm{j} \omega) \leqslant 0$ for all $\omega$, and (without loss of generality) $\arg G(0)=\arg w_{1}(0)=\arg w_{2}(0)=0$.

The argument function is usually only defined modulo $2 \pi$. To obtain a well-defined argument function, we make the argument function continuous for all $\omega$ and fix the value of the argument at $\omega=0$. This is always possible here since zeroes and poles on the imaginary axis are not allowed. This is for convenience, though it is still possible otherwise, to avoid having to consider small indentations in the left or right complex half plane like in Nyquist D-contours.

Now the optimization problem can be formulated. Given a transfer function $G$ and weights $w_{1}, w_{2}$ that satisfy Assumption 2, and an integer $m$, find $\phi$ and $G_{\mathrm{a}}$ such that

$$
\begin{gathered}
\min _{\phi, G_{\mathrm{a}}} \phi \quad \text { s.t. } \\
-\phi+\arg w_{2}(\mathrm{j} \omega)<\arg G_{\mathrm{a}}(\mathrm{j} \omega)-\arg G(\mathrm{j} \omega)<\phi-\arg w_{1}(\mathrm{j} \omega) \text { for all } \omega \\
0 \leqslant \phi<\frac{\pi}{2}, \quad 2 \phi-\pi \leqslant \arg w_{1}(\mathrm{j} \omega)+\arg w_{2}(\mathrm{j} \omega) \text { for all } \omega \\
G_{\mathrm{a}} \in Q_{m}
\end{gathered}
$$

This problem is almost completely analogous to magnitude approximation (5)-(7). The constraint (31) has a clear interpretation in a Bode diagram. The constant $\phi$ plays here the role that $\gamma$ did in magnitude approximation. If $\phi>0$ it means that the phase bounds are relaxed, see Figure 2. Hence, in the minimization problem we will find the approximation $G_{\mathrm{a}}$ that satisfies the tightest possible bounds. It will be shown in Section 3.1 that (30)-(33) becomes a quasiconvex minimization problem.

There are two major differences between magnitude and phase approximation. These are contained in (32). Firstly, there is an upper bound on the relaxation $\phi$, and secondly, there is a constraint on the weights $w_{1}, w_{2}$ and $\phi$. Hence, whereas we always find solutions in the magnitude case (for sufficiently large $\gamma$ ), in the phase case, there are problems that have no feasible solution. In case of an infeasible problem, one needs to increase the model order $m$, or modify the weights. 


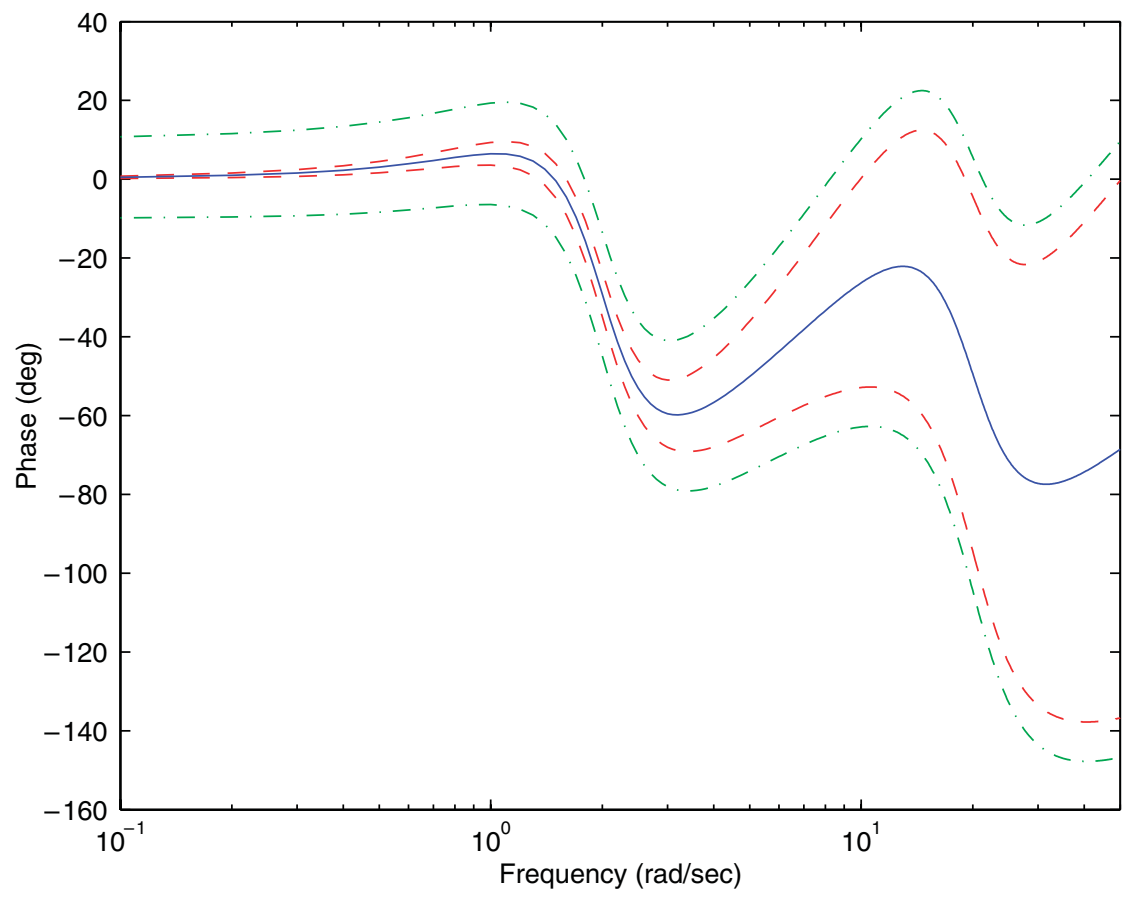

Figure 2. The interpretation of the weights $w_{1}, w_{2}$, and the constant $\phi$. The full line represents the model $G$, the dashed lines represent the bounds with $\phi=0$, and the dash-dotted lines the relaxed bounds with $\phi=10^{\circ}$. The argument of the approximation $G_{\mathrm{a}}$ should be within the bounds with the smallest possible $\phi$.

\section{Remark 8}

The assumption $2 \phi-\pi \leqslant \arg w_{1}(\mathrm{j} \omega)+\arg w_{2}(\mathrm{j} \omega) \Leftrightarrow\left(\phi-\arg w_{1}(\mathrm{j} \omega)\right)+\left(\phi-\arg w_{2}(\mathrm{j} \omega)\right) \leqslant \pi$ means that the relaxed bounds in Figure 2 are never allowed to be more than 180 degrees apart. The reason for this assumption is that we will use Cartesian co-ordinates to obtain a convex feasibility problem. The Cartesian co-ordinates simplify the parametrization and ensure that the arguments of $G$ and $G_{\mathrm{a}}$ are always on the same branch. Of course, this also restrict us from going into different branches.

\section{Remark 9}

If the weights $\arg w_{1}$ and $\arg w_{2}$ are chosen to be zero in (30)-(33), a solution to (2) is obtained. It should also be clear why we assume $\arg w_{1} \leqslant 0$ and $\arg w_{2} \leqslant 0$, since otherwise with $\phi=0$ a perfect approximation $\arg G(\mathrm{j} \omega)=\arg G_{\mathrm{a}}(\mathrm{j} \omega)$ would not be allowed.

\subsection{Parametrization and the semidefinite program}

In this section, we show how (30)-(33) is translated into a semidefinite program. We also show how the program may be solved when used for model reduction, i.e. using state-space data, and system identification, i.e. using frequency samples. First we introduce the parametrization that is used to obtain $G_{\mathrm{a}}$. The parametrization is less direct in this case, but it is shown in Theorem 5 that it is appropriate. Let $m$ be a positive integer (which will turn out to be related to the order 
of $G_{\mathrm{a}}$ ) and define

$$
T_{m}(s)=1+\sum_{i=1}^{m} t_{i} s^{i}
$$

where $\left\{t_{i}\right\}$ are the $m$ decision variables. The polynomial $T_{m}$ is used to define the real functions $c_{m, 1}(\omega), c_{m, 2}(\omega), d_{m, 1}(\omega), d_{m, 2}(\omega)$ as

$$
\begin{gathered}
\left(w_{1} G^{*} T_{m}\right)(\mathrm{j} \omega)=: c_{m, 1}(\omega)+\mathrm{j} d_{m, 1}(\omega) \\
\left(w_{2} T_{m}^{*} G\right)(\mathrm{j} \omega)=: c_{m, 2}(\omega)+\mathrm{j} d_{m, 2}(\omega)
\end{gathered}
$$

Notice that these functions are linear in the decision variables $\left\{t_{i}\right\}$, since $G, w_{1}, w_{2}$ are fixed. These functions $c_{m, 1}, c_{m, 2}, d_{m, 1}, d_{m, 2}$ are used to find $\left\{t_{i}\right\}$, which are used to construct $T_{m}(s)$, which in turn gives $G_{\mathrm{a}}(s)$. This is explained in the following theorem that is the phase approximation counterpart of Theorem 3 .

\section{Theorem 5}

Assume that $G, w_{1}, w_{2}$ satisfy Assumption 2, that $\phi$ is fixed and satisfies (32), and $m$ is a fixed positive integer. Then there is a $G_{\mathrm{a}} \in Q_{m}$ that satisfies (31) if and only if there are $c_{m, 1}, c_{m, 2}, d_{m, 1}$, $d_{m, 2}$, defined by (35), that satisfy

$$
d_{m, 1}(\omega)<(\tan \phi) c_{m, 1}(\omega)
$$

and

$$
d_{m, 2}(\omega)<(\tan \phi) c_{m, 2}(\omega)
$$

for all $\omega$.

Proof

First notice that (36) is equivalent to

$$
\phi-\pi<\arg \left(w_{1} G^{*} T_{m}\right)(\mathrm{j} \omega)<\phi
$$

and

$$
-\phi<\arg \left(w_{2}^{*} G^{*} T_{m}\right)(\mathrm{j} \omega)<\pi-\phi
$$

for all $\omega$, since $\phi \in[0, \pi / 2)$. By our continuity assumption on the argument functions together with the assumption $2 \phi-\pi \leqslant \arg w_{1}(\mathrm{j} \omega)+\arg w_{2}(\mathrm{j} \omega)$ this is equivalent to

$$
-\phi+\arg w_{2}(\mathrm{j} \omega)<\arg T_{m}(\mathrm{j} \omega)-\arg G(\mathrm{j} \omega)<\phi-\arg w_{1}(\mathrm{j} \omega)
$$

for all $\omega$.

We now prove the 'only if' part of the theorem. $G_{\mathrm{a}}$ fulfils (31) by assumption. Since $G_{\mathrm{a}} \in Q_{m}$ it can be factorized as

$$
G_{\mathrm{a}}(s)=G_{\mathrm{a}}(0) \frac{\prod_{i=1}^{m-p}\left(1-s / z_{i}\right)}{\prod_{i=1}^{p}\left(1-s / p_{i}\right)}, \quad p \in[0, m]
$$

where $z_{i}, p_{i} \in \mathbb{C}_{-}$. If we define $T_{m}(s)=\prod_{i=1}^{m-p}\left(1-s / z_{i}\right) \prod_{i=1}^{p}\left(1+s / p_{i}^{*}\right)$ then $\arg G_{\mathrm{a}}(\mathrm{j} \omega)=\arg T_{m}$ (j $\omega$ ) for all $\omega$ and $T_{m}$ can be expressed in the form (34). With the equivalence of (36) and (37), the result follows. 
The 'if' part of the proof follows if we notice that a solution $T_{m}$ can be factorized as

$$
T_{m}(s)=\prod_{i=1}^{m-p}\left(1-s / z_{i}^{-}\right) \prod_{i=1}^{p}\left(1-s / z_{i}^{+}\right)
$$

where $z_{i}^{+} \in \mathbb{C}_{+}$and $z_{i}^{-} \in \mathbb{C}_{-}$. Notice that $T_{m}$ cannot have any zeroes on the imaginary axis because then $c_{m, 1}(\omega)=d_{m, 1}(\omega)=c_{m, 2}(\omega)=c_{m, 2}(\omega)=0$ for some frequency $\omega$, and that violates (36). A $G_{\mathrm{a}} \in Q_{m}$ with the desired properties is now constructed as

$$
G_{\mathrm{a}}(s)=\frac{\prod_{i=1}^{m-p}\left(1-s / z_{i}^{-}\right)}{\prod_{i=1}^{p}\left(1+s /\left(z_{i}^{+}\right)^{*}\right)}
$$

This concludes the proof.

To ensure a proper approximation $G_{\mathrm{a}}$ we must see to it that $p \geqslant m-p$. This can, for example, be ensured by the following lemma.

\section{Lemma 6}

Assume that $G \in R H_{\infty}$ is minimum phase and has relative degree $\eta$. Assume furthermore that

$$
\left|\arg G_{\mathrm{a}}(\mathrm{j} \omega)-\arg G(\mathrm{j} \omega)\right|<\pi / 2 \quad \text { as } \omega \rightarrow \infty
$$

Then $G_{\mathrm{a}} \in Q_{m}$ constructed by (38)-(39) has relative degree $\eta, G_{\mathrm{a}} \in R_{(\eta+m) / 2} H_{\infty}$, and $m=\eta+2 n$, for some integer $n \geqslant 0$.

\section{Proof}

The argument of $G$ and $G_{\mathrm{a}}$ at infinity are equal to $-\pi \eta / 2$ and $-\pi(2 p-m) / 2$. By the assumption we have that $|-\eta+2 p-m|<1$, and because all numbers are integers we conclude that $2 p-m=\eta$. Hence, the relative degree of (39) is $\eta$. We have that $p=(\eta+m) / 2$, and $G_{\mathrm{a}} \in R_{p} H_{\infty}$. The last statement follows since $m=\eta+2(m-p)$ and $m-p \geqslant 0$ since it is the number of zeroes of $G_{\mathrm{a}}$.

Remark 10 (Selection of $m$ and McMillan degree of $G_{\mathrm{a}}$ )

Notice that Lemma 6 says that $m \geqslant \eta$ and it only makes sense to seek approximations $T_{m}$ of degree equal to the relative degree of $G$ plus $0,2,4,6, \ldots$ Furthermore, each time $m$ is increased by two, the McMillan degree of the sought approximation $G_{\mathrm{a}}$ increases by one.

One can easily enforce (40) by proper choice of the weight functions $w_{1}$ and $w_{2}$. If one does not prescribe bounds such that (40) holds, the obtained approximation $G_{\mathrm{a}}$ may have different relative degree than $G$ has. This is usually undesirable.

Theorem 5 shows that for fixed $\phi$ and $m,(31)-(33)$ is a convex feasibility problem, since (36) is linear in the unknowns $\left\{t_{i}\right\}$. Again, this implies that (30)-(33) is a quasiconvex minimization problem. There are infinitely many constraints in (36) because of the dependence on $\omega$. Just as in the magnitude case, the KYP lemma can be used to tackle this. An alternative, since we here only consider SISO transfer functions, is to use sum of squares decompositions (SOS) [20]. In the system identification situation, things are easier and we only need to solve a linear program. The details are discussed next. 
3.1.1. Phase model reduction and sum of squares programs. Here we assume that the model $G$ and weights $w_{1}, w_{2}$ are given in transfer function form. Then there are polynomials $A_{1}, A_{2}, B_{1}, B_{2}$ such that

$$
\left(w_{1} G^{*}\right)(\mathrm{j} \omega)=\frac{B_{1}(\mathrm{j} \omega)}{A_{1}(\mathrm{j} \omega)}, \quad\left(w_{2} G\right)(\mathrm{j} \omega)=\frac{B_{2}(\mathrm{j} \omega)}{A_{2}(\mathrm{j} \omega)}
$$

From (35) it follows that there are real polynomials $\bar{c}_{m, 1}, \bar{c}_{m, 2}, \bar{d}_{m, 1}, \bar{d}_{m, 2}$, linear in $\left\{t_{i}\right\}$, such that

$$
\begin{gathered}
c_{m, 1}(\omega)=\frac{\operatorname{Re}\left(B_{1} A_{1}^{*} T_{m}\right)(\mathrm{j} \omega)}{\left|A_{1}(\mathrm{j} \omega)\right|^{2}}=\frac{\bar{c}_{m, 1}(\omega)}{\left|A_{1}(\mathrm{j} \omega)\right|^{2}}, \quad d_{m, 1}(\omega)=\frac{\operatorname{Im}\left(B_{1} A_{1}^{*} T_{m}\right)(\mathrm{j} \omega)}{\left|A_{1}(\mathrm{j} \omega)\right|^{2}}=\frac{\bar{d}_{m, 1}(\omega)}{\left|A_{1}(\mathrm{j} \omega)\right|^{2}} \\
c_{m, 2}(\omega)=\frac{\operatorname{Re}\left(B_{2} A_{2}^{*} T_{m}^{*}\right)(\mathrm{j} \omega)}{\left|A_{2}(\mathrm{j} \omega)\right|^{2}}=\frac{\bar{c}_{m, 2}(\omega)}{\left|A_{2}(\mathrm{j} \omega)\right|^{2}}, \quad d_{m, 2}(\omega)=\frac{\operatorname{Im}\left(B_{2} A_{2}^{*} T_{m}^{*}\right)(\mathrm{j} \omega)}{\left|A_{2}(\mathrm{j} \omega)\right|^{2}}=\frac{\bar{d}_{m, 2}(\omega)}{\left|A_{2}(\mathrm{j} \omega)\right|^{2}}
\end{gathered}
$$

and since $\left|A_{1}(\mathrm{j} \omega)\right|>0,\left|A_{2}(\mathrm{j} \omega)\right|>0$, (36) can equivalently be formulated as

$$
\bar{p}_{m, 1}(\omega, \phi):=(\tan \phi) \bar{c}_{m, 1}(\omega)-\bar{d}_{m, 1}(\omega) \geqslant \varepsilon
$$

and

$$
\bar{p}_{m, 2}(\omega, \phi):=(\tan \phi) \bar{c}_{m, 2}(\omega)-\bar{d}_{m, 2}(\omega) \geqslant \varepsilon
$$

for all $\omega$ and some $\varepsilon>0$. This condition means that we need to find $\left\{t_{i}\right\}$ such that the polynomials $\bar{p}_{m, 1}(\omega, \phi)=\bar{p}_{m, 1}\left(\omega, \phi,\left\{t_{i}\right\}\right)$ and $\bar{p}_{m, 2}(\omega, \phi)=\bar{p}_{m, 2}\left(\omega, \phi,\left\{t_{i}\right\}\right)$ are positive for all $\omega$. By construction, $\bar{c}_{m, 1}$ and $\bar{c}_{m, 2}$ only contain monomials of even degree, and $\bar{d}_{m, 1}$ and $\bar{d}_{m, 2}$ only contain monomials of odd degree. A necessary condition for (41) to hold is that the degrees of $\bar{p}_{m, 1}$ and $\bar{p}_{m, 2}$ are even. This is another motivation for the restriction on the selection of $m$ that was seen in Lemma 6. Let us denote the even degree of $\bar{p}_{m, 1}$ with $2 n_{1}$ and the even degree of $\bar{p}_{m, 2}$ with $2 n_{2}$.

It is a fact that a polynomial in one variable is non-negative if and only if it can be written as a sum of polynomial squares [23]. This equivalence can be utilized to reformulate (41) into the following set of LMIs [20] which is often called a sum of squares program:

$$
\begin{gathered}
\text { Find }\left\{t_{i}\right\}, \quad M_{k}=M_{k}^{\mathrm{T}} \in \mathbb{R}^{n_{k} \times n_{k}} \geqslant 0, \quad \varepsilon>0 \quad \text { subject to } \\
\left(\begin{array}{c}
\omega^{n_{k}} \\
\omega^{n_{k}-1} \\
\vdots \\
1
\end{array}\right) M_{k}^{\mathrm{T}}\left(\begin{array}{c}
\omega^{n_{k}} \\
\omega^{n_{k}-1} \\
\vdots \\
1
\end{array}\right)=\bar{p}_{m, k}\left(\omega, \phi,\left\{t_{i}\right\}\right)-\varepsilon \text { for all } \omega, k=1,2
\end{gathered}
$$

This feasibility problem is readily solved by standard LMI solvers in MATLAB, and there are special toolboxes designed for the task [22,24].

The following bisection algorithm can now be used to solve (30)-(33). 
Algorithm 2 (Phase model reduction)

Input: transfer functions $G(s), w_{1}(s), w_{2}(s)$ that satisfy Assumption 2, an integer $m$, and tolerance $\varepsilon_{\text {tol }}>0$. Output: if the problem has a feasible solution, $G_{\mathrm{a}} \in Q_{m}$ that solves (30)-(33) with accuracy $\varepsilon_{\text {tol }}$.

1. If $\phi_{\max }=\sup _{\omega}\left(\arg w_{1}(\mathrm{j} \omega)+\arg w_{2}(\mathrm{j} \omega)+\pi\right) / 2 \geqslant \pi / 2$, the problem has no feasible solution.

2. If (42) is feasible with $\phi=\phi_{u}=0$, goto 7 .

3. If (43) is not feasible with $\phi=\phi_{u}=\phi_{\max }$, the problem has no feasible solution, else $\phi_{l}=0$.

4. Put $\phi_{t}=\left(\phi_{u}+\phi_{l}\right) / 2$.

5. If (42) is feasible with $\phi=\phi_{t}$, then $\phi_{u}=\phi_{t}$, else $\phi_{l}=\phi_{t}$.

6. If $\phi_{u}-\phi_{l}>\varepsilon_{\mathrm{tol}}$, goto 4 .

7. From the feasible solution associated with $\phi_{u}$, compute $G_{\mathrm{a}} \in Q_{m}$ as in (38)-(39).

3.1.2. Phase system identification and finite number of data points. Just as in the magnitude case, the minimization problem becomes simpler when there are only finitely many frequency points $\left\{\omega_{k}\right\}$ to consider. In the phase system identification case, (31) reduces to finding $\left\{t_{i}\right\}$ such that

$$
\bar{p}_{m, 1}\left(\omega_{i}, \phi,\left\{t_{i}\right\}\right)>0
$$

and

$$
\bar{p}_{m, 2}\left(\omega_{i}, \phi,\left\{t_{i}\right\}\right)>0
$$

for all $\omega_{i} \in\left\{\omega_{k}\right\}$. This is a linear program, and it can be solved with, for example, MATLAB for very large sets of frequencies $\left\{\omega_{k}\right\}$ and parameters $\left\{t_{i}\right\}$. Once a solution is found, $G_{\mathrm{a}} \in Q_{m}$ is constructed as before in (38)-(39).

\subsection{Relative $H_{\infty}$ error bounds}

In this section, we state a result about how systems that have similar phases also can have a small relative $H_{\infty}$ error. Just as in Section 2.2, the result is based on bounds on the Hilbert transform operator restricted in its application to rational transfer functions. These are derived in [10].

The phase function is a way of representing the argument. We call $\Phi_{G}$ the phase function of $G$ when

$$
\Phi_{G}(\mathrm{j} \omega):=\frac{G(\mathrm{j} \omega)}{G(\mathrm{j} \omega)^{*}}=\exp (2 \mathrm{j} \arg G(\mathrm{j} \omega))
$$

Notice that phase functions are all-pass functions. We have the following counterpart to Theorem 4.

\section{Theorem 7}

Assume that $G, G_{\mathrm{a}} \in R L_{\infty}$ have no zeroes on the imaginary axis and that $\varepsilon \in[0,2)$. Then

$$
-\arcsin \frac{\varepsilon}{2} \leqslant \arg G_{\mathrm{a}}(\mathrm{j} \omega)-\arg G(\mathrm{j} \omega) \leqslant \arcsin \frac{\varepsilon}{2} \text { for all } \omega
$$

is equivalent to

$$
\left\|\Phi_{G_{\mathrm{a}}}-\Phi_{G}\right\|_{\infty} \leqslant \varepsilon
$$


If furthermore $\left(G_{\mathrm{a}}^{-1} G\right),\left(G^{-1} G_{\mathrm{a}}\right) \in R_{n} H_{\infty},\left(G_{\mathrm{a}}^{-1} G\right)(\infty)=1, \varepsilon<1 /(2 n)$, then (44) implies

$$
\begin{aligned}
& \left\|G^{-1}\left(G-G_{\mathrm{a}}\right)\right\|_{\infty} \leqslant 2 n \varepsilon /(1-2 n \varepsilon) \\
& \left\|G_{\mathrm{a}}^{-1}\left(G-G_{\mathrm{a}}\right)\right\|_{\infty} \leqslant 2 n \varepsilon /(1-2 n \varepsilon)
\end{aligned}
$$

\section{Proof}

We first prove the equivalence of (44) and (45). We have that $\left\|\Phi_{G_{\mathrm{a}}}-\Phi_{G}\right\|_{\infty}=\left\|\Phi_{G_{\mathrm{a}}} \Phi_{G}^{*}-1\right\|_{\infty} \leqslant \varepsilon$. Since all arguments are continuous this is equivalent to $\exp \left(2 \mathrm{j}\left(\arg G_{\mathrm{a}}-\arg G\right)(\mathrm{j} \omega)\right)-1 \mid \leqslant \varepsilon$ for all $\omega$. This is equivalent to $2 \sin \left|\left(\arg G_{\mathrm{a}}-\arg G\right)(\mathrm{j} \omega)\right| \leqslant \varepsilon$, for all $\omega$, which concludes the first part of the proof.

Next we prove that (44) implies (46). The result follows from [10, Theorem 4.1] if we use $v_{1}=G_{\mathrm{a}}$ and $v_{2}=G$. The bound (45) and the assumption $\varepsilon<1 /(2 n)$ then gives $\left\|v_{1} / v_{1}^{*}-v_{2} / v_{2}^{*}\right\|_{\infty} \leqslant \varepsilon<1 /(2 n)$. The bounds (46) now follow directly from (4.12a) in [10, Theorem 4.1].

Hence, not only do we get a good match of the phase if the phase model reduction problem is solved, we may also get guarantees that the transfer functions $G$ and $G_{\text {a }}$ are close. This is of course provided that the minimum phase property $\left(G_{\mathrm{a}}^{-1} G\right),\left(G^{-1} G_{\mathrm{a}}\right) \in R_{n} H_{\infty}$ holds.

\section{EXAMPLES}

MATLAB together with SeDuMi [21] and YALMIP [22] are used for all the examples.

Example 1 (Model reduction-magnitude)

Using Algorithm 1 on the 35th order model

$$
G(s)=\prod_{k=1}^{35} \frac{s+2 k}{s+2 k-1}
$$

with $m=2$ and $w_{1}=w_{2}=1$ we obtain an approximation

$$
G_{\mathrm{a}}(s)=\frac{1.035 s^{2}+42.09 s+138.2}{s^{2}+12.63 s+13.15}
$$

with $\gamma=0.1\left(\varepsilon_{\mathrm{tol}}=0.01\right)$. Hence, according to Corollary 2 , the magnitude curves will be no more than $0.38 \mathrm{~dB}$ apart for all $\omega$. That the bound is tight can be seen in Figure 3. For comparison we also apply four other standard model reduction methods to the example. We use Hankel norm approximation, balanced truncation, singular perturbation on the balanced realization, and balanced stochastic truncation, see [4]. The relative error $\mid\left(G(\mathrm{j} \omega)-G_{\mathrm{a}}(\mathrm{j} \omega)\right) /$ $G(\mathrm{j} \omega) \mid$ is plotted in Figure 4 for the five different approximations. As can be seen, the approximation obtained with Algorithm 1 offers the best compromise amongst all methods in this example. In this case, the a priori bound (27) gives $\left\|G^{-1}\left(G-G_{\mathrm{a}}\right)\right\|_{\infty} \leqslant 16.8 \mathrm{~dB}$ which is a very conservative bound if we compare with the real error in Figure 4. One would perhaps expect this given the list of inequalities used to derive the bound (27). 


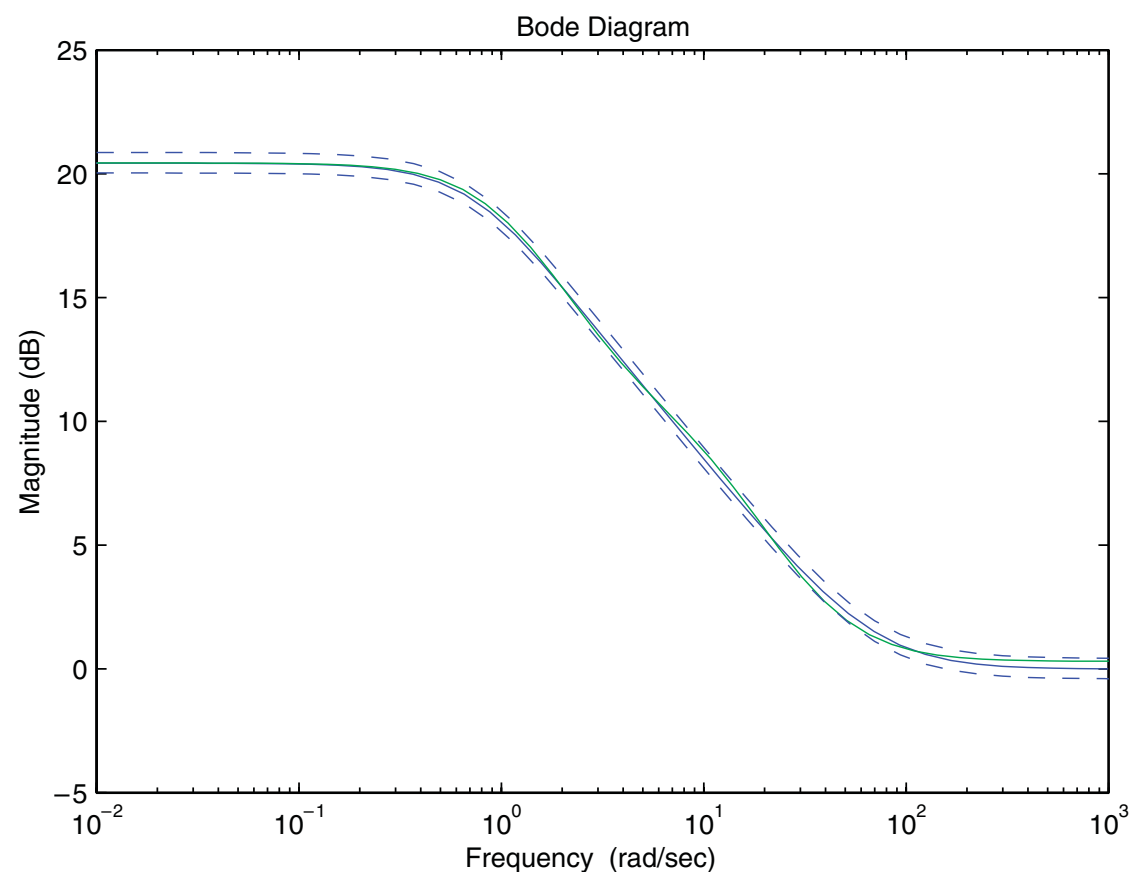

Figure 3. The model $G$ and the second-order approximation $G_{\text {a }}$ obtained in Example 1. The uniform bounds are plotted with dashed lines. Here $\gamma=0.1$, which gives an error bound of $0.38 \mathrm{~dB}$.

Example 2 (System identification-magnitude)

Here we use frequency samples of a 104th order transfer function

$$
G(s)=\frac{(s+2)^{2}}{s^{2}+0.4 s+4} \cdot \frac{(s+20)^{2}}{s^{2}+12 s+400} \cdot \prod_{k=1}^{100} \frac{s+2 k}{s+2 k-1}
$$

on a grid $\left\{\omega_{k}\right\}=\{0.1,0.2, \ldots, 50\}$. Furthermore, we are only interested in a good approximation for low frequencies and choose the weights

$$
w_{1}(s)=\frac{1}{s / 20+1} \quad \text { and } \quad w_{2}(s)=\frac{1}{(s / 10+1)^{2}}
$$

and put $m=2$. We use the magnitude system identification method described in Section 2.1.2. There is no solution with $\gamma=0$ for the posed problem, so the bounds are relaxed and $\gamma$ is increased to $0.051\left(\varepsilon_{\mathrm{tol}}=0.001\right)$ when a solution is found. The approximation and the relaxed bounds are shown in Figure 5. The obtained approximation is

$$
G_{\mathrm{a}}(s)=\frac{7.434 s^{2}+32.96 s+70.59}{s^{2}+0.3792 s+4.071}
$$

The approximation satisfies the bounds in Corollary 2 on the grid $\left\{\omega_{k}\right\}$. In this example, it is hard to compare the result to any other method. Already model reduction with symmetric 


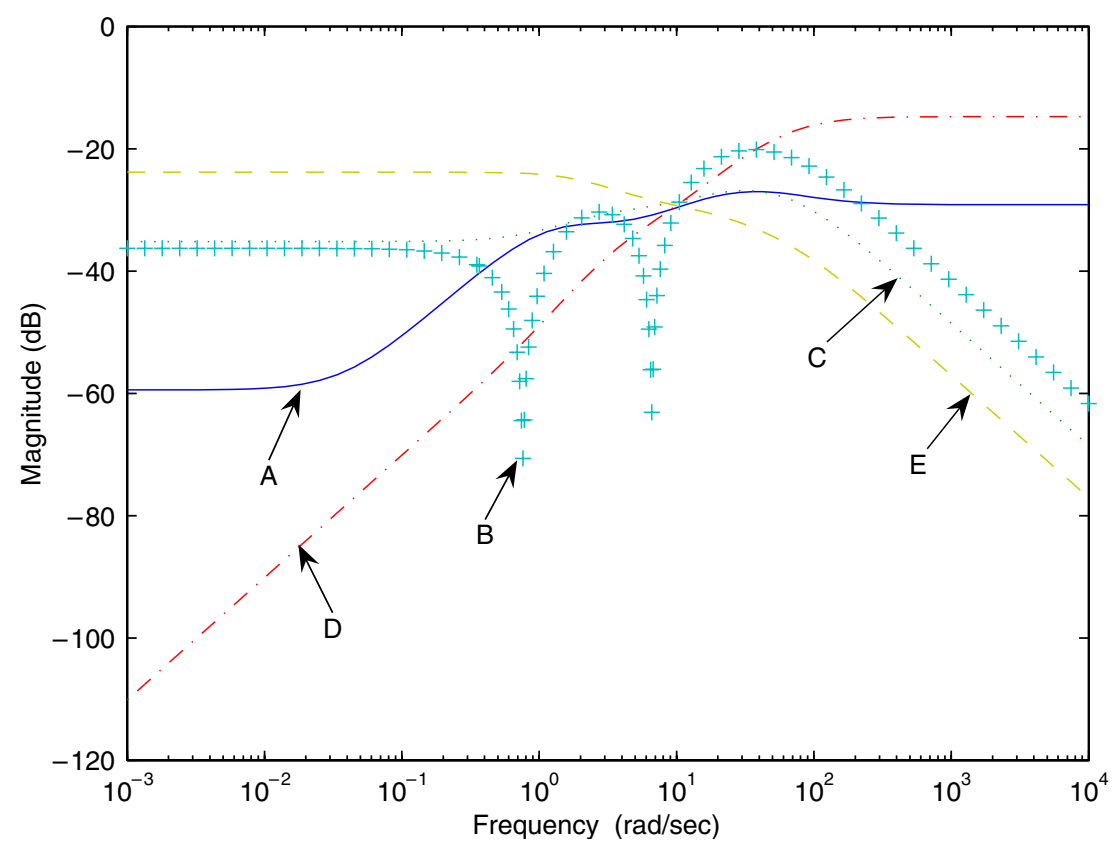

Figure 4. The relative error $\left|\left(G(\mathrm{j} \omega)-G_{\mathrm{a}}(\mathrm{j} \omega)\right) / G(\mathrm{j} \omega)\right|$ is plotted for the approximations in Example 1. The different second-order approximations are Algorithm 1 (A), Hankel norm approximation (B), balanced truncation (C), singular perturbation of the balanced realization (D), and balanced stochastic truncation (E).

weights is a difficult problem, see [4]. Furthermore, these available methods require a state-space model of $G$ which should not be required for system identification.

\section{Example 3 (System identification-phase)}

Here we use the phase system identification method to construct a low-pass filter that has linear phase for low frequencies. It is often desirable to have filters with linear phase, since these do not distort the signal (compare with a time delay). In discrete time, one can easily construct linear phase Finite Impulse Response (FIR) filters. However, here we are concerned with continuoustime rational filters. We will therefore fit a rational transfer function $G_{\mathrm{a}}(s)$ to frequency samples $G\left(\mathrm{j} \omega_{k}\right)$ generated by a discrete-time linear phase FIR filter

$$
G(\mathrm{j} \omega)=\sum_{l=0}^{5} c_{l} \mathrm{e}^{-l \pi \mathrm{j} \omega}
$$

with cut-off frequency $0.5 \mathrm{rad} / \mathrm{s}$ and $\left\{\omega_{k}\right\}=\{0,0.01, \ldots, 0.7\}$. The coefficients $\left\{c_{l}\right\}$ are given by fir $1(5,0.5)$ in Matlab. We choose to look for $G_{\mathrm{a}}$ in $Q_{5}(m=5)$. Then the minimum relaxation becomes $\phi=0.013 \mathrm{rad}\left(\varepsilon_{\mathrm{tol}}=2 \times 10^{-4} \mathrm{rad}\right)$, and $G_{\mathrm{a}}$ becomes

$$
G_{\mathrm{a}}(s)=\frac{0.01472}{s^{5}+1.011 s^{4}+0.9101 s^{3}+0.4047 s^{2}+0.1193 s+0.01472}
$$




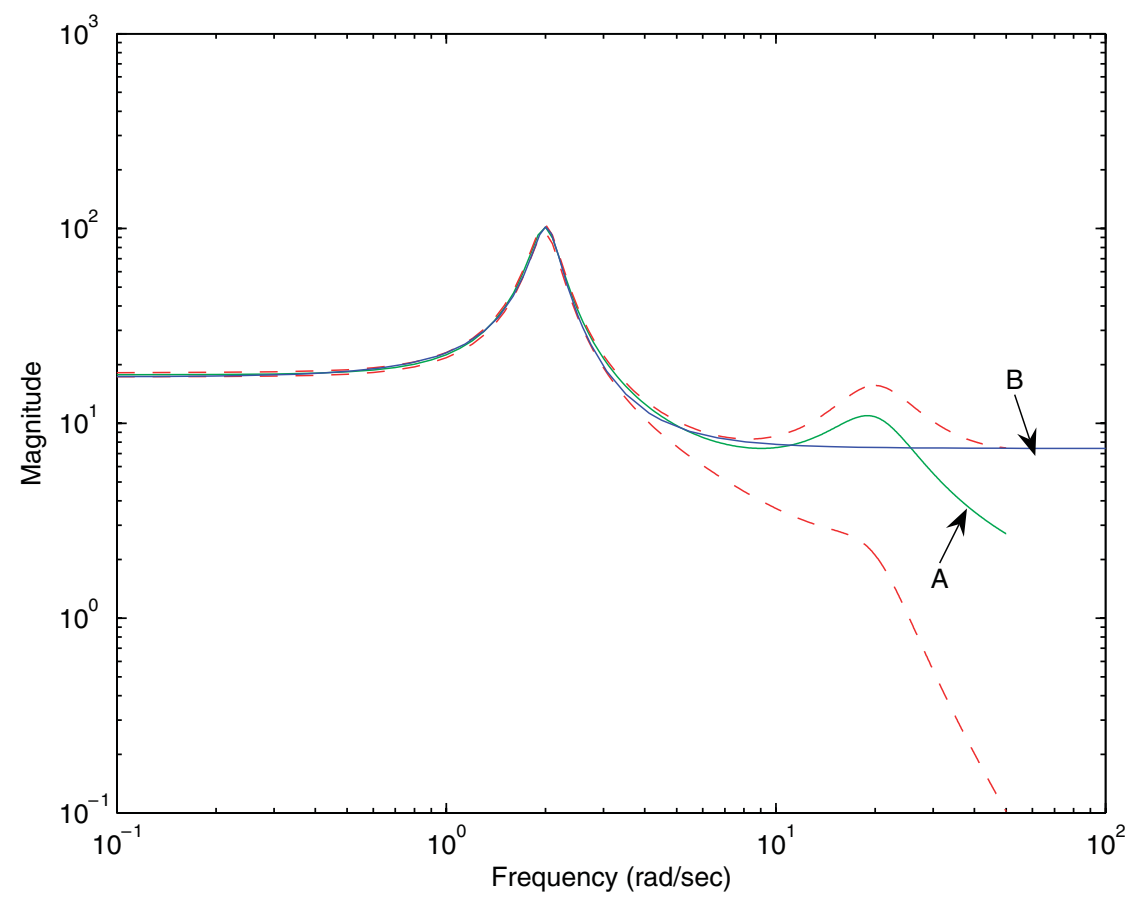

Figure 5. The frequency data $G\left(\mathrm{j} \omega_{k}\right)$ (A) together with the relaxed bounds $w_{1}, w_{2}$ (dashed lines, $\gamma=0.051$ ) and the approximation $G_{\mathrm{a}}(\mathrm{j} \omega)(\mathrm{B})$ from Example 2 .

As can be seen in Figure 6, the phase of the filter $G_{\mathrm{a}}$ is very close to the phase of $G$ up to $0.7 \mathrm{rad} / \mathrm{s}$ and the cut-off frequency is close to $0.5 \mathrm{rad} / \mathrm{s}$. The largest phase error on $\left\{\omega_{k}\right\}$ is $0.013 \mathrm{rad}$.

For comparison, we compute two 5 th order Bessel filters, $G_{\mathrm{B}}^{(1)}$ and $G_{\mathrm{B}}^{(2)}$, using besself $(5,0.5)$ and besself $(5,0.7)$ in Matlab. Bessel filters are often used for design of analog filters with approximately linear phase. In this case, the filters have (by design) almost linear phase up to 0.5 and $0.7 \mathrm{rad} / \mathrm{s}$, respectively. The Bessel filters are quite different from $G_{\mathrm{a}}$, as can be seen in Figure 6. The filter $G_{\mathrm{B}}^{(1)}$ has almost the same (linear) phase behaviour as $G$ and $G_{\mathrm{a}}$ up to $0.5 \mathrm{rad} / \mathrm{s}$ but has a lower cut-off frequency. The filter $G_{\mathrm{B}}^{(2)}$ has almost linear phase up to $0.7 \mathrm{rad} / \mathrm{s}$, just as $G_{\mathrm{a}}$, but has less phase lag and a higher cut-off frequency than the other filters. Hence, by using the phase system identification method, we can design low-pass filters with almost linear phase but with other characteristics than standard Bessel filters.

\section{Example 4 (System identification-comparison with [7])}

Here we illustrate the differences between the magnitude and the phase system identification methods presented in this paper, and the method developed in [7]. The main difference between the methods is the approximation criteria. In this paper, we minimize the magnitude and the phase error, whereas in [7], the additive $L_{\infty}$ error is minimized. As we shall see, this results in approximations with different properties. 

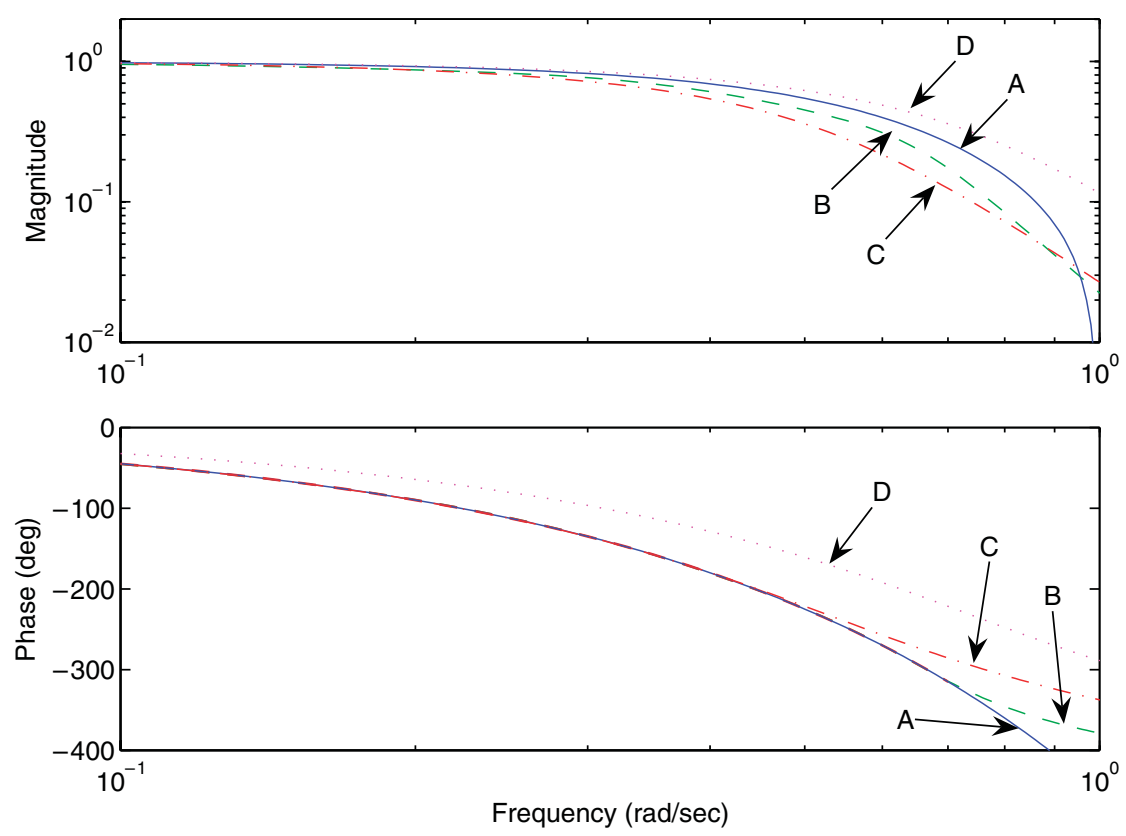

Figure 6. The frequency data $G\left(\mathrm{j} \omega_{k}\right)(\mathrm{A})$, the 5th-order phase approximation $G_{\mathrm{a}}(\mathrm{B})$, and the 5 th-order Bessel filters $G_{\mathrm{B}}^{(1)}(\mathrm{C})$ and $G_{\mathrm{B}}^{(2)}(\mathrm{D})$ from Example 3. The low-pass filter $G_{\mathrm{a}}$, designed with the phase system identification method, has almost linear phase behaviour up to frequency $0.7 \mathrm{rad} / \mathrm{s}$. The Bessel filters have almost linear phase up to 0.5 and $0.7 \mathrm{rad} / \mathrm{s}$, respectively, but have other cut-off frequencies than $G$ and $G_{\mathrm{a}}$.

We use the model $G$ in (47) and the grid $\left\{\omega_{k}\right\}=\{0.1,0.11, \ldots, 10\}$, and $w_{1}=w_{2}=1$. The method in [7] gives

$$
G_{\mathrm{a}}^{(1)}=\frac{6.477 s^{2}+35.05 s+68.04}{s^{2}+0.4016 s+4.032}
$$

with $\gamma=0.88$, magnitude system identification gives

$$
G_{\mathrm{a}}^{(2)}=\frac{6.863 s^{2}+35.37 s+69.81}{s^{2}+0.4052 s+4.076}
$$

with $\gamma=0.07$, and phase system identification gives

$$
G_{\mathrm{a}}^{(3)}=\frac{6.347 s^{2}+36.19 s+72.39}{s^{2}+0.4151 s+4.087}
$$

with $\phi=0.04$ rad. Four different error plots are shown in Figure 7. In the top left figure, the additive error $\left|G-G_{\mathrm{a}}\right|$ is plotted. As expected, $G_{\mathrm{a}}^{(1)}$ has the overall best performance in this case, and $G_{\mathrm{a}}^{(2)}, G_{\mathrm{a}}^{(3)}$ have relatively large peaks. In the top right figure, the relative error $\left|\left(G-G_{\mathrm{a}}\right) / G\right|$ is plotted. Here the phase approximation $G_{\mathrm{a}}^{(3)}$ has the overall best performance, and $G_{\mathrm{a}}^{(1)}, G_{\mathrm{a}}^{(2)}$ are comparable, even though $G_{\mathrm{a}}^{(2)}$ has the highest peak. In the bottom left figure, the magnitude error $|\log | G|-\log | G_{\mathrm{a}} \|$ is plotted together with the minimal bound $\gamma=0.07$. Here the approximation $G_{\mathrm{a}}^{(2)}$ has the smallest overall error. The phase approximation $G_{\mathrm{a}}^{(3)}$ has the largest magnitude error. In the bottom right figure, the phase error $\left|\arg G-\arg G_{\mathrm{a}}\right|$ is plotted together 

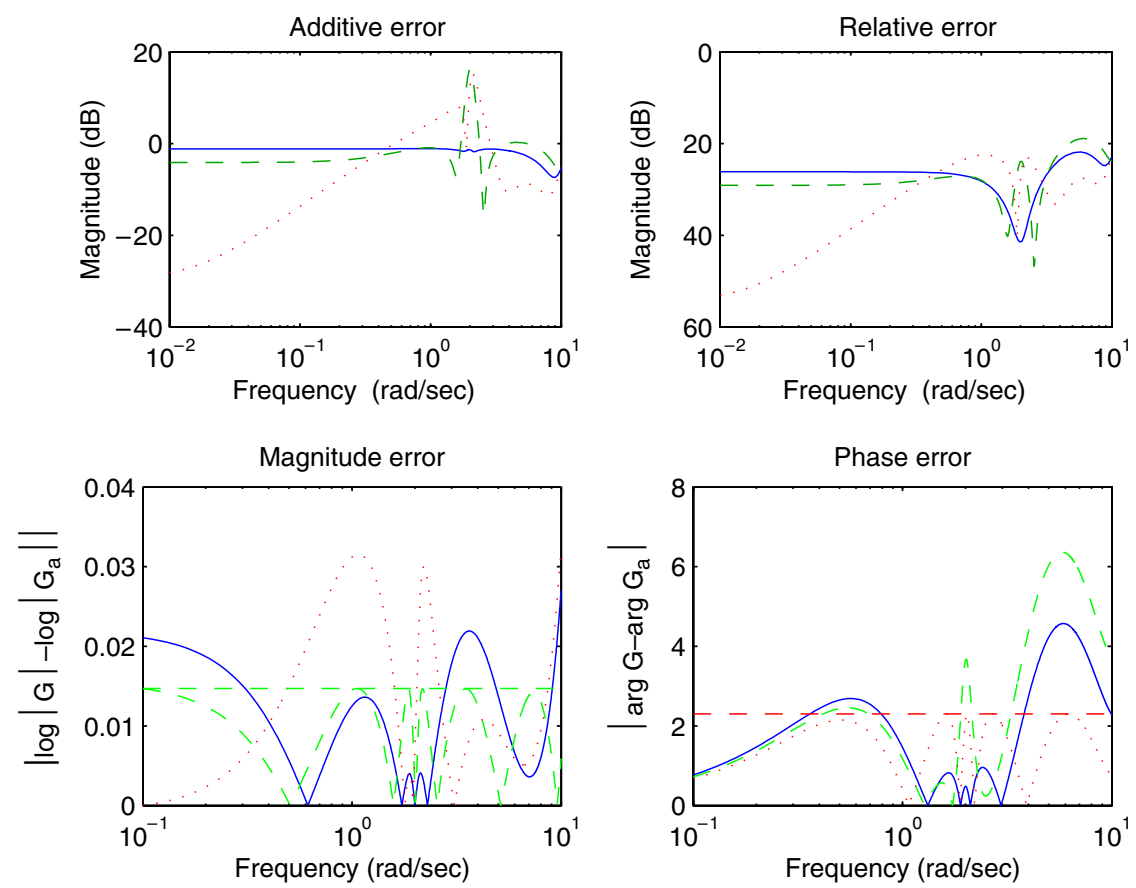

Figure 7. Error plots for $G_{\mathrm{a}}^{(1)}$ (solid), the magnitude approximation $G_{\mathrm{a}}^{(2)}$ (dashed), and the phase approximation $G_{\mathrm{a}}^{(3)}$ (dotted). The minimum possible upper bounds on the magnitude error $(0.5 \log (1+\gamma)=$ $0.015)$ and the phase error $\left(\phi=2.3^{\circ}\right)$ are also included in the lower plots. These bound are tight for the magnitude and phase approximations.

with the minimal bound $\phi=0.04 \mathrm{rad}\left(=2.3^{\circ}\right)$. Here the approximation $G_{\mathrm{a}}^{(3)}$ has the smallest overall error, and the magnitude approximation $G_{\mathrm{a}}^{(2)}$ has the largest error.

To summarize, not surprisingly, there is a case where each method performs best. It depends on the error criterion used. In the relative error case, which neither method directly minimizes, the phase approximation $G_{\mathrm{a}}^{(3)}$ has the overall best performance in this example. The method used for $G_{\mathrm{a}}^{(1)}$ uses both magnitude and phase data in its approximation, and it offers in some of the cases a compromise between the methods developed in this paper. Computationally, $G_{\mathrm{a}}^{(1)}$ was the most expensive approximation to obtain in this example. This was because two optimization runs were used in each iteration (one for the denominator and one for the numerator of $G_{\mathrm{a}}^{(1)}$ ), and because each sample $G\left(\mathrm{j} \omega_{k}\right)$ gave rise to a $2 \times 2$ LMI. For $G_{\mathrm{a}}^{(2)}$ and $G_{\mathrm{a}}^{(3)}$, only one optimization run was used in each iteration, and only scalar inequalities were needed. Scalar inequalities are used also in [7], but there a dedicated cutting plane method is described. For this example, we only used a standard LMI solver [21,22].

\section{CONCLUSIONS}

We have shown how one can match transfer functions of given degree to magnitude and phase data using standard LMI solvers. We considered both the case where a continuum of data was 
available (model reduction case), and when only samples of data (system identification case) were available. In the magnitude case, we dealt with both SISO and MIMO systems, whereas in the phase case only SISO systems were considered. We also showed that under the assumption that the systems were stable and minimum phase, systems that have bounded magnitude or phase error also satisfy a simple a priori bound on the relative $H_{\infty}$ error. We illustrated the results in Bode diagrams, where the results are easily understood, and with four examples. The examples showed that the results are useful in several areas, such as, in model reduction, system identification, and in signal processing.

An interesting topic for future research is to match magnitude and phase data over complementary frequency intervals. For this, methods from [25] could be useful. Furthermore, how to deal with phase approximation for MIMO systems is still an open issue. There are also many applications where the results could be useful. For example, in the design of band-pass linear phase filters.

\section{ACKNOWLEDGEMENTS}

This work was supported by the Australian Research Council under Discovery-Projects Grant DP0342683, and by National ICT Australia Ltd., which is funded through the Australian Government's Backing Australia's Ability Initiative. Parts of this work were performed during a month's visit of the first author at the Australian National University.

\section{REFERENCES}

1. Boyd SP, El Ghaoui L, Feron E, Balakrishnan V. Linear Matrix Inequalities in System and Control Theory. SIAM: Philadelphia, PA, 1997.

2. Dullerud GE, Paganini F. A Course in Robust Control Theory-A Convex Approach. Springer: Berlin, 2000.

3. Moore BC. Principal component analysis in linear systems: controllability, observability, and model reduction. IEEE Transactions on Automatic Control 1981; 26(1):17-32.

4. Obinata G, Anderson BDO. Model Reduction for Control System Design. Springer: London, U.K., 2001.

5. Glover K. All optimal Hankel-norm approximations of linear multivariable systems and their $L_{\infty}$-error bounds. International Journal of Control 1984; 39:1115-1193.

6. Megretski A. Optimal model order reduction for maximal real part norms. In Directions in Mathematical Systems Theory and Optimization, Rantzer A, Byrnes C (eds), Lecture Notes in Control and Information Sciences, vol. 286. Springer: Berlin, 2003; 217-226.

7. Sou KC, Megretski A, Daniel L. A quasi-convex optimization approach to parametrized model order reduction. Proceedings of Design Automation Conference, Anaheim, CA, U.S.A., June 2005.

8. MacFarlane AGJ, Postlethwaite I. The generalized Nyquist stability criterion and multivariable root loci. International Journal of Control 1977; 25:81-127.

9. Opdenacker P, Jonckheere EA. State space approach to approximation by phase matching. Modelling, Identification and Robust Control, Stockholm, Sweden, June 1986; 349-362.

10. Anderson BDO, Green M. Hilbert transform and gain/phase error bounds for rational functions. IEEE Transactions on Circuits and Systems 1988; 35(5):528-535.

11. Sandberg H, Lanzon A, Anderson BDO. Transfer function approximation and identification using magnitude and phase criteria. In Proceedings of the 17th International Symposium on Mathematical Theory of Networks and Systems, Kyoto, Japan, 24-28 July 2006; 250-256.

12. Boyd S, Vandenberghe L. Convex Optimization. Cambridge University Press: Cambridge, 2004.

13. Horn RA, Johnson CR. Matrix Analysis. Cambridge University Press: Cambridge, 1990.

14. Zhou K, Doyle JC, Glover K. Robust and Optimal Control. Prentice-Hall: Upper Saddle River, NJ, 1996.

15. Anderson BDO. An algebraic solution to the spectral factorization problem. IEEE Transactions on Automatic Control 1967; 12(4):410-414.

16. Yakubovich VA. The solution of certain matrix inequalities in automatic control theory. Soviet Mathematics Doklady 1962; 3:620-623. 
17. Yakubovich VA. The solution of certain matrix inequalities encountered in nonlinear control theory. Soviet Mathematics Doklady 1964; 5:652-656.

18. Yakubovich VA, Leonov GA, Gelig AKh. Stability of Stationary Sets in Control Systems with Discontinuous Nonlinearities. World Scientific: Singapore, 2004.

19. Rantzer A. On the Kalman-Yakubovich-Popov lemma. Systems and Control Letters 1996; 28(1):7-10.

20. Parrilo PA. Semidefinite programming relaxations for semialgebraic problems. Mathematical Programming 2003; 96(2):293-320.

21. Sturm JF. Using SeDuMi 1.02, a MATLAB toolbox for optimization over symmetric cones. Optimization Methods and Software 1999; 11-12:625-653. Available from http://fewcal.kub.nl/sturm/software/sedumi.html

22. Löfberg J. YALMIP: a toolbox for modeling and optimization in MATLAB. Proceedings of the CACSD Conference, Taipei, Taiwan, 2004. Available from http://control.ee.ethz.ch/ joloef/yalmip.php

23. Reznick B. Some concrete aspects of Hilbert's 17th problem. Contemporary Mathematics 2000; 253:251-272.

24. Prajna S, Papachristodoulou A, Seiler P, Parrilo PA. SOSTOOLS: Sum of Squares Optimization Toolbox for MATLAB, 2004

25. Parker PJ, Anderson BDO, Araki M. Construction of mixed Hilbert transforms. Proceedings of ISSPA 90, Signal Processing, Theories, Implementations and Applications, Gold Coast, Australia, August 1990; 823-828. 